\title{
Analytic structure and power-series expansion of the Jost function for the two-dimensional problem
}

\author{
S. A. Rakityansky ${ }^{1) *}$ and N. Elander ${ }^{2}$ \\ 1) Dept. of Physics, University of Pretoria, Pretoria 0002, South Africa \\ 2) Div.of Molecular Physics, Dept. of Physics, Stockholm University, \\ Stockholm, SE-106 91, Sweden
}

December 7, 2018

\begin{abstract}
For a two-dimensional quantum mechanical problem, we obtain a generalized powerseries expansion of the $S$-matrix that can be done near an arbitrary point on the Riemann surface of the energy, similarly to the standard effective range expansion. In order to do this, we consider the Jost-function and analytically factorize its momentum dependence that causes the Jost function to be a multi-valued function. The remaining single-valued function of the energy is then expanded in the power-series near an arbitrary point in the complex energy plane. A systematic and accurate procedure has been developed for calculating the expansion coefficients. This makes it possible to obtain a semi-analytic expression for the Jost-function (and therefore for the S-matrix) near an arbitrary point on the Riemann surface and use it, for example, to locate the spectral points (bound and resonant states) as the S-matrix poles. The method is applied to a model simlar to those used in the theory of quantum dots.
\end{abstract}

PACS number(s): 03.65.Nk, 03.65.Ge, 24.30.Gd

*e-mail: rakitsa@up.ac.za 


\section{Introduction}

It is probable that an average reader of this journal perceives the one- and two-dimensional problems as simplified toy models of quantum mechanics. Although such an attitude has its roots in the standard courses of quantum mechanics, this perception is far from being adequate. First of all, modern nano-technology allows us to fabricate the microscopic quantum devices that behave and can be described as one- or two-dimensional. The two-dimensional tunneling of particles play an important role in superconductive tunnel junctions and even in some biological molecules [1]. Besides that, the corresponding quantum mechanical problems are not mathematically simple as one may think. Indeed, in contrast to the motion of a particle in three-dimensional space, the one-dimensional motion of the same particle on an infinite line is inherently a two-channel problem, where the channels are the left and right halves of the line (see, for example, Refs. [2,3]). As far as the two-dimensional scattering is concerned, its amplitude as a function of the energy has not a square-root but a logarithmic branching point (see Refs. [4-10] as well as the subsequent sections of the present paper). Therefore from both pure mathematical and practical points of view, the two-dimensional quantum problem is worthwhile to consider.

In the present paper, we focus on solving the two-dimensional problem with the help of power series that are similar to famous effective-range expansion (where $\hbar k$ and $\delta_{0}$ are the collision momentum and the $S$-wave phase-shift)

$$
k \cot \delta_{0}(k)=-\frac{1}{a}+\frac{1}{2} r_{0} k^{2}-P r_{0}^{3} k^{4}+Q r_{0}^{5} k^{6}+\cdots,
$$

in terms of the so called scattering length $a$, effective radius $r_{0}$, etc., introduced long ago in nuclear physics [11].

As we already mentioned, the energy dependent functions of the two-dimensional problem have a logarithmic branching point at the threshold. As a result there is a controversy concerning the two-dimensional analog of Eq. (1). Some authors [6-8, 12] define the scattering length $a^{\prime}$ by including it in the logarithmic term,

$$
\cot \delta_{0}(k)=\frac{2}{\pi}\left(\gamma+\ln \frac{k a^{\prime}}{2}\right)+\frac{r_{0}^{2}}{2 \pi} k^{2}+\mathcal{O}\left(k^{4}\right),
$$

(here $\gamma$ is the Euler's constant) while the others [4,5] try to preserve the traditional form of the right hand side of Eq. (1) and move the logarithmic term to the left hand side,

$$
\cot \delta_{0}(k)-\frac{2}{\pi}\left(\gamma+\ln \frac{k}{2}\right)=-\frac{2}{a^{\prime \prime}}+\mathcal{O}\left(k^{2}\right),
$$


where $\ln a^{\prime}=-\pi / a^{\prime \prime}$.

We look at this problem from a more general point of view. What is actually done in the original effective range expansion (1) is the constructing of the function $R(E)=k \cot \delta_{0}(k)$ in which the square-root branching of $k \sim \pm \sqrt{E}$ at the threshold is compensated by exactly the same branching of $\delta_{0}(k) \sim \pm \sqrt{E}$. As a result the function $R(E)$ does not have branching points and is a single-valued analytic function of the energy $E \sim k^{2}$, and therefore can be expanded in a convergent series $R(E)=a_{0}+a_{1} E+a_{2} E^{2}+\cdots$, which is given by Eq. (1).

From this reasoning a next logical step immediately follows: the function $R(E)$ can be expanded in a more general power series $R(E)=b_{0}+b_{1}\left(E-E_{0}\right)+b_{2}\left(E-E_{0}\right)^{2}+\cdots$ around an arbitrary complex energy $E_{0}$ within the domain of its analyticity. In Refs. [13, 14], we realized this idea for the three-dimensional single-channel and multi-channel problems. In doing this, instead of using $R(E)$, we expanded the analytic single-valued parts of the Jost functions (or Jost matrices in the multi-channel case) after explicit separation of the factors that are responsible for the branching.

In the present paper, we use the same approach as in Refs. [13,14] to obtain similar expansions of the Jost functions for the two-dimensional problem. First, we analyze the analytic structure of the Jost functions and split them in the single-valued and logarithmically branching parts. Then, we derive a set of differential equations that determine the single-valued parts. And finally, we look for the solutions of these equations in the form of power series of the energy. The series (2) and (3) together with simple recipes for calculating any number of their expansion coefficients, are easily obtained from our more general expansions that are done around an arbitrary complex point $E_{0}$. Using two-dimensional model potential related to quantum dot theory, we numerically demonstrate the efficiency and accuracy of the proposed method.

\section{Jost function}

Radial part $u_{\ell}$ of the wave function describing the motion of a particle of mass $\mu$ with the energy $E$ in a circularly symmetric two-dimensional potential $U(r)=\hbar^{2} V(r) / 2 \mu$ obeys the differential equation (a review of the partial-wave analysis for the two-dimensional scattering is given in the Appendix (A)

$$
\left[\frac{d^{2}}{d r^{2}}+k^{2}-\frac{\lambda(\lambda+1)}{r^{2}}-V(r)\right] u_{\ell}(E, r)=0,
$$


where $\ell=\lambda+1 / 2$ is the angular momentum and $\lambda=-1 / 2,1 / 2,3 / 2, \ldots$. At large distances, where $V(r) \rightarrow 0$, the radial equation simplifies

$$
\left[\frac{d^{2}}{d r^{2}}+k^{2}-\frac{\lambda(\lambda+1)}{r^{2}}\right] u_{\ell}(E, r) \approx 0, \quad r \rightarrow \infty .
$$

This is the Riccati-Bessel equation. As its two linearly independent solutions, we can choose either the Riccat-Bessel and Riccati-Neumann functions $j_{\lambda}(k r)$ and $y_{\lambda}(k r)$, or the two RiccatiHankel functions $h_{\lambda}^{( \pm)}(k r)$. Any other solution of Eq. (5) is a superposition of the two linearly independent solutions. In particular, we can write the asymptotics of the physical wave function as a linear combination of the Riccati-Hankel functions,

$$
u_{\ell}(E, r) \underset{r \rightarrow \infty}{\longrightarrow} f_{\ell}^{(\mathrm{in})}(E) h_{\ell-1 / 2}^{(-)}(k r)+f_{\ell}^{(\mathrm{out})}(E) h_{\ell-1 / 2}^{(+)}(k r)
$$

where the energy-dependent combination coefficients $f_{\ell}^{\text {(in/out) }}(E)$ are called the Jost functions. When $r \rightarrow \infty$, the Riccati-Hankel functions represent the incoming and outgoing circular waves. Indeed,

$$
h_{\ell-1 / 2}^{( \pm)}(k r)=\sqrt{\frac{\pi k r}{2}} H_{\ell}^{( \pm)}(k r) \underset{|z| \rightarrow \infty}{\longrightarrow} e^{ \pm i(k r-\ell \pi / 2-\pi / 4)}=\mp i e^{ \pm i(k r-\lambda \pi / 2)}
$$

where $H_{\ell}^{( \pm)}(z)$ are the cylindrical Hankel functions. The Jost functions $f_{\ell}^{(\text {in/out })}(E)$ are therefore the asymptotic amplitudes of the incoming and outgoing waves. Since the flux of the particles is conserving, for real $E$ we have $\left|f_{\ell}^{(\text {in) }}(E)\right|=\left|f_{\ell}^{\text {(out) }}(E)\right|$. Actually, these two functions are related to each other at different complex values of $E$ as well. Some of such symmetry properties can be established using the semi-analytic structure of them that is derived in the subsequent sections. It can also be shown that the partial wave $S$-matrix is the ratio of these functions

$$
s_{\ell}(E)=\frac{f_{\ell}^{(\text {out })}(E)}{f_{\ell}^{(\text {in })}(E)}
$$

and that zeros of the Jost function $f_{\ell}^{(\mathrm{in})}(E)$ are the discrete spectral points $\mathcal{E}_{n}$,

$$
f_{\ell}^{(\mathrm{in})}\left(\mathcal{E}_{n}\right)=0
$$

i.e. the bound and resonant states of the system. 


\section{Transformation of the radial equation}

Our goal is to establish the analytic structure of the Jost function, i.e. to find such an expression for it where all possible nonanalytic dependencies on the energy are given explicitly. This can be done if we transform the second-order radial equation (4) into an equivalent set of first-order equations.

The transformation is done using a method which is known in the theory of differential equations as the variation parameters method [15, 16]. Following this method, we look for the unknown function $u_{\ell}(E, r)$ in the form similar to its asymptotics (6), but with the combination coefficients being new unknown functions of $r$,

$$
u_{\ell}(E, r)=F_{\ell}^{(\mathrm{in})}(E, r) h_{\ell-1 / 2}^{(-)}(k r)+F_{\ell}^{(\mathrm{out})}(E, r) h_{\ell-1 / 2}^{(+)}(k r),
$$

where $F_{\ell}^{(\text {in/out })}(E, r)$ are the new unknown functions. Since instead of one unknown function, we introduce two of them, they cannot be independent of each other. In principle, we can impose any reasonable condition relating them. Looking at the asymptotics (6), we see that

$$
f_{\ell}^{(\text {in })}(E)=\lim _{r \rightarrow \infty} F_{\ell}^{(\text {in })}(E, r), \quad f_{\ell}^{(\text {out })}(E)=\lim _{r \rightarrow \infty} F_{\ell}^{(\text {out })}(E, r) .
$$

Therefore at large distances our new functions become constants, and we should have

$$
\left[\partial_{r} F_{\ell}^{(\mathrm{in})}\right] h_{\lambda}^{(-)}(k r)+\left[\partial_{r} F_{\ell}^{(\text {out })}\right] h_{\lambda}^{(+)}(k r)=0 .
$$

As the additional condition imposed on these functions, we demand that the relation (12) is valid not only at large $r$ but at all distances. In the variation parameters method this condition is known as the Lagrange condition. In our case this condition makes $F_{\ell}^{(\text {in/out })}(E, r)$ to be the Jost functions for the potential which is cut-off at the radius $r$ (in the spirit of the variable-phase approach).

Substituting the ansatz (10) into the radial equation (4) and using the Lagrange condition (12) together with known Wronskian of the Riccati-Hankel functions,

$$
h_{\lambda}^{(-)}(k r) \partial_{r} h_{\lambda}^{(+)}(k r)-h_{\lambda}^{(+)}(k r) \partial_{r} h_{\lambda}^{(-)}(k r)=2 i k,
$$

we obtain a set of two first-order equations which are equivalent to the original radial equation (4),

$$
\begin{aligned}
\partial_{r} F_{\ell}^{(\mathrm{in})} & =-\frac{1}{2 i k} h_{\lambda}^{(+)} V\left[F_{\ell}^{(\mathrm{in})} h_{\lambda}^{(-)}+F_{\ell}^{(\mathrm{out})} h_{\lambda}^{(+)}\right], \\
\partial_{r} F_{\ell}^{(\mathrm{out})} & =+\frac{1}{2 i k} h_{\lambda}^{(-)} V\left[F_{\ell}^{(\mathrm{in})} h_{\lambda}^{(-)}+F_{\ell}^{(\mathrm{out})} h_{\lambda}^{(+)}\right] .
\end{aligned}
$$


The boundary conditions for these equations follow from the requirement that the wave function must be regular everywhere. In particular, this means that $u_{\ell}(E, 0)=0$. It seems that this is not the case because both $h_{\lambda}^{(+)}(k r)$ and $h_{\lambda}^{(-)}(k r)$ that are present in the expression (10), are singular at $r=0$. Their singularities, however, can cancel each other,

$$
h_{\lambda}^{(+)}(z)+h_{\lambda}^{(-)}(z)=2 j_{\lambda}(z)
$$

if they are superimposed with a same coefficient. This can be achieved if both $F_{\ell}^{(\mathrm{in})}(E, r)$ and $F_{\ell}^{\text {(out) }}(E, r)$ have the same value at $r=0$,

$$
F_{\ell}^{(\text {in })}(E, 0)=F_{\ell}^{\text {(out) }}(E, 0) .
$$

Their common value at $r=0$ determines the overall normalization of the wave function and therefore can be chosen arbitrarily. To be consistent, we chose it to be $1 / 2$, which makes $u_{\ell}(E, r)$ to behave near the origin exactly as the Riccati-Bessel function and thus the solution with the boundary conditions

$$
F_{\ell}^{(\mathrm{in})}(E, 0)=F_{\ell}^{(\text {out })}(E, 0)=\frac{1}{2}
$$

is what is called the regular solution in the theory of three-dimensional scattering.

For our goal of expressing the non-analytic dependencies of the Jost functions in an explicit form, it is more convenient to re-write the ansatz (10) in terms of the Riccati-Bessel and Riccati-Neumann functions,

$$
u_{\ell}(E, r)=A_{\ell}(E, r) j_{\lambda}(k r)-B_{\ell}(E, r) y_{\lambda}(k r),
$$

and to obtain the corresponding equations for the unknown functions $A_{\ell}(E, r)$ and $B_{\ell}(E, r)$. Since

$$
h_{\lambda}^{( \pm)}(z)=j_{\lambda}(z) \pm i y_{\lambda}(z)
$$

this is most simply achieved by making the following linear combinations of Eqs. (14, 15)

$$
\begin{aligned}
& A_{\ell}(E, r)=F_{\ell}^{(\text {in })}(E, r)+F_{\ell}^{(\text {out })}(E, r), \\
& B_{\ell}(E, r)=i\left[F_{\ell}^{(\text {in })}(E, r)-F_{\ell}^{(\text {out })}(E, r)\right] .
\end{aligned}
$$

This gives

$$
\begin{aligned}
& \partial_{r} A_{\ell}=-\frac{1}{k} y_{\lambda} V\left(A_{\ell} j_{\lambda}-B_{\ell} y_{\lambda}\right) \\
& \partial_{r} B_{\ell}=-\frac{1}{k} j_{\lambda} V\left(A_{\ell} j_{\lambda}-B_{\ell} y_{\lambda}\right)
\end{aligned}
$$


with the boundary conditions

$$
A_{\ell}(E, 0)=1, \quad B_{\ell}(E, 0)=0 .
$$

\section{Complex rotation}

Suppose that the potential $V(r)$ is cut off at certain radius $r=R$, then the right-hand sides of the sets of equations (14]15) or (22] 23) vanish at $r>R$ and thus the derivatives on the left-hand sides of these equations become zero, i.e. the functions $F_{\ell}^{(\text {in/out })}$ or $A_{\ell}$ and $B_{\ell}$ do not change beyond this point. Therefore, in the spirit of the variable phase approach, the functions $F_{\ell}^{\text {(in/out) }}(E, r)$ are the Jost functions for the potential which is cut off at the point $r$. Generally speaking, when the potential asymptotically vanishes at large distances, we should expect the convergence of the limits (11).

Therefore, the Jost functions can be calculated by numerical integration of the differential equations (1415) or (2223) from $r=0$ up to a sufficiently large radius $R$ where the limits (11) are reached within a required accuracy. This works perfectly for real values of the energy $E$. However, when we consider complex energies (for example, in search for resonances), a technical difficulty arises. This difficulty is caused by the asymptotic behavior (7) of the Riccati-Hankel functions.

When $k$ is complex, either $h_{\lambda}^{(+)}(k r)$ or $h_{\lambda}^{(-)}(k r)$ exponentially diverges, depending on the sign of $\operatorname{Im} k$. As a result, either the first or the second of the equations (14 15) does not give a numerically convergent solution. This difficulty is circumvented by using the deformed integration path shown in figure 1. Instead of integrating the differential equations along the real axis from $r=0$ to $r=R$, we can reach the final point via the intermediate point $r=R^{\prime}$ in the complex plane. Moreover, we can safely ignore the $\operatorname{arc} R^{\prime} R$ since the potential is practically zero at that distance.

Such a complex rotation helps because the asymptotic behavior (divergent or convergent) of the functions $h_{\lambda}^{( \pm)}(k r)$ is determined by the sign of $\operatorname{Im} k$. For a given $k=|k| e^{i \phi}$, we can always find such a rotation angle $\theta$ in $r=|r| e^{-i \theta}$ that the product $k r=|k r| e^{i(\phi-\theta)}$ has either positive or negative (or even zero) imaginary part. Various technical details of using complex rotation in calculating the Jost functions and Jost matrices can be found in [2,3, 13, 14, 18,-25]. 


\section{$5 \quad$ Explicit separation of the non-analytic factors}

In order to establish the analytic structure of the Jost functions, we need to have a closer look at the structure of the Riccati-Bessel and Riccat-Neumann functions. The following expressions for them (they can be derived using formulae 9.1.2, 9.1.10, and 9.1.11 of Ref. [17]) are the most useful for this

$$
\begin{aligned}
& j_{\lambda}(k r)=k^{\lambda+1} \sum_{n=0}^{\infty} k^{2 n} f_{n}^{(\lambda)}(r), \\
& y_{\lambda}(k r)=k^{-\lambda} \sum_{n=0}^{\infty} k^{2 n} g_{n}^{(\lambda)}(r)+h(k) j_{\lambda}(k r),
\end{aligned}
$$

where

$$
f_{n}^{(\lambda)}(r)=\frac{\sqrt{\pi}(-1)^{n}}{n ! \Gamma(n+\lambda+3 / 2)}\left(\frac{r}{2}\right)^{2 n+\lambda+1}, \quad \text { for any } \lambda .
$$

If $\lambda$ is integer then the expansion of $y_{\lambda}(k r)$ is also simple:

$$
\begin{aligned}
g_{n}^{(\lambda)}(r) & =\frac{\sqrt{\pi}(-1)^{n+\lambda+1}}{n ! \Gamma(n-\lambda+1 / 2)}\left(\frac{r}{2}\right)^{2 n-\lambda}, \\
h(k) & =0 .
\end{aligned}
$$

However, for a half-integer $\lambda$, we have a more difficult case:

$$
\begin{aligned}
g_{n}^{(\lambda)}(r)= \begin{cases}-\frac{(\lambda-n-1 / 2) !}{\sqrt{\pi} n !}\left(\frac{r}{2}\right)^{2 n-\lambda} & , 0 \leqslant n \leqslant \lambda-\frac{1}{2} \\
\frac{2}{\pi} \ln \left(\frac{r}{R}\right) f_{n-\lambda-\frac{1}{2}}^{(\lambda)}(r)- & \\
-\frac{(-1)^{n-\lambda-\frac{1}{2}}\left[\psi(n+1)+\psi\left(n-\lambda+\frac{1}{2}\right)\right]}{\sqrt{\pi} n !\left(n-\lambda-\frac{1}{2}\right) !}\left(\frac{r}{2}\right)^{2 n-\lambda}, & \lambda+\frac{1}{2} \leqslant n<\infty\end{cases} \\
h(k)=\frac{2}{\pi} \ln \left(\frac{k R}{2}\right),
\end{aligned}
$$

where $R$ is an arbitrary number (in the units of length). It is arbitrary because any increase or decrease of $h(k)$ cased by the change of $R$ is compensated by the corresponding change in the first term of Eq. (30). The parameter $R$ is introduced to separate the $r$ and $k$ dependencies 
in the original term containing $\ln (k r / 2)=\ln (k R / 2)+\ln (r / R)$ and to have dimensionless products under the logarithm. In practical calculations the parameter $R$ can always be taken as the unit of the length, i.e. $R=1$. The $\psi$-function in Eq. (30) is defined as follows [17]

$$
\psi(n)=\frac{\Gamma^{\prime}(n)}{\Gamma(n)}= \begin{cases}-\gamma & n=1 \\ -\gamma+\sum_{m=1}^{n-1} m^{-1} & , n \geqslant 2\end{cases}
$$

where $\gamma=0.577 \ldots$ is the Euler constant.

Eqs. (25, 26) represent the Riccati-Bessel and Riccati-Neuman functions in the form of infinite series. Each term of these series is a product of a function depending on $k$ and another function depending on $r$, i.e. the $k$ and $r$ dependencies are given in a separable form.

What do the above formulae tell us about the Jost functions? The functions $j_{\lambda}(k r)$ and $y_{\lambda}(k r)$ are involved in the coefficients of the differential equations (22/23) that determine the Jost functions. This means that the Jost functions are not single valued functions of the energy. Indeed, for each choice of $E$, we have two possible values of the momentum

$$
k= \pm \sqrt{\frac{2 \mu E}{\hbar^{2}}} .
$$

The index $\lambda=-1 / 2,1 / 2,3 / 2, \ldots$ of the Riccati functions is a half-integer. This means that the differential equations involve such multi-valued functions as square-root and logarithm of the momentum.

Therefore the Jost functions are defined on a complicated Riemann surface and the threshold point $E=0$ is a branching point of this surface. It would be desirable to find an expression for the Jost functions in terms of the powers of $\sqrt{k}$, the logarithmic function $h(k)$, and some entire single valued functions of $E$. In order to do this, we notice that the series in Eqs. (25,26) involve only even powers of $k$, i.e. the powers of the energy $k^{2}=2 \mu E / \hbar^{2}$. Since for any finite $r$ these series are absolutely and uniformly convergent on the whole complex plane of $E$, they define some entire functions, i.e.

$$
\begin{aligned}
j_{\lambda}(k r) & =k^{\lambda+1} \tilde{j}_{\lambda}(E, r), \\
y_{\lambda}(k r) & =k^{-\lambda} \tilde{y}_{\lambda}(E, r)+k^{\lambda+1} h(k) \tilde{j}_{\lambda}(E, r),
\end{aligned}
$$


where the "tilded" functions

$$
\begin{aligned}
& \tilde{j}_{\lambda}(E, r)=\sum_{n=0}^{\infty}\left(\frac{2 \mu E}{\hbar^{2}}\right)^{n} f_{n}^{(\lambda)}(r), \\
& \tilde{y}_{\lambda}(E, r)=\sum_{n=0}^{\infty}\left(\frac{2 \mu E}{\hbar^{2}}\right)^{n} g_{n}^{(\lambda)}(r),
\end{aligned}
$$

are single-valued entire functions of complex variable $E$.

Let us find a similar structure for the functions $A_{\ell}(E, r)$ and $B_{\ell}(E, r)$ and through them for $F_{\ell}^{(\text {in/out })}(E, r)$. For this, we replace the set of equations (22) 23) with their linear combinations. Namely, we multiply Eq. (23) by $h(k)$ and subtract the result from Eq. (22); and as the second equation, we take Eq. (23) multiplied by $k^{-(2 \lambda+1)}$. As a result, we obtain:

$$
\begin{aligned}
\partial_{r}\left(A_{\ell}-h B_{\ell}\right) & =-\frac{1}{k}\left(y_{\lambda}-h j_{\lambda}\right) V\left(A_{\ell} j_{\lambda}-B_{\ell} y_{\lambda}\right), \\
\partial_{r} k^{-(2 \lambda+1)} B_{\ell} & =-k^{-2(\lambda+1)} j_{\lambda} V\left(A_{\ell} j_{\lambda}-B_{\ell} y_{\lambda}\right) .
\end{aligned}
$$

Now, taking into account Eqs. (33, 34), we see that

$$
y_{\lambda}-h j_{\lambda}=k^{-\lambda} \tilde{y}_{\lambda}+k^{\lambda+1} h \tilde{j}_{\lambda}-k^{\lambda+1} h \tilde{j}_{\lambda}=k^{-\lambda} \tilde{y}_{\lambda}
$$

and

$$
\begin{aligned}
A_{\ell} j_{\lambda}-B_{\ell} y_{\lambda} & =A_{\ell} k^{\lambda+1} \tilde{j}_{\lambda}-B_{\ell} k^{-\lambda} \tilde{y}_{\lambda}-B_{\ell} k^{\lambda+1} h \tilde{j}_{\lambda} \\
& =k^{\lambda+1}\left(A_{\ell}-h B_{\ell}\right) \tilde{j}_{\lambda}-k^{-\lambda} B_{\ell} \tilde{y}_{\lambda} .
\end{aligned}
$$

Substituting these expressions into Eqs. (37, 38), we have

$$
\begin{aligned}
\partial_{r}\left(A_{\ell}-h B_{\ell}\right) & =-k^{-(\lambda+1)} \tilde{y}_{\lambda} V\left[k^{\lambda+1}\left(A_{\ell}-h B_{\ell}\right) \tilde{j}_{\lambda}-k^{-\lambda} B_{\ell} \tilde{y}_{\lambda}\right], \\
\partial_{r} k^{-(2 \lambda+1)} B_{\ell} & =-k^{-(\lambda+1)} \tilde{j}_{\lambda} V\left[k^{\lambda+1}\left(A_{\ell}-h B_{\ell}\right) \tilde{j}_{\lambda}-k^{-\lambda} B_{\ell} \tilde{y}_{\lambda}\right] .
\end{aligned}
$$

If we introduce the "tilded" functions

$$
\begin{aligned}
\tilde{A}_{\ell}(E, r) & \equiv A_{\ell}(E, r)-h(k) B_{\ell}(E, k) \\
\tilde{B}_{\ell}(E, r) & \equiv k^{-(2 \lambda+1)} B_{\ell}(E, r)
\end{aligned}
$$


then Eqs. (39, 40) assume the following form

$$
\begin{aligned}
& \partial_{r} \tilde{A}_{\ell}=-\tilde{y}_{\lambda} V\left(\tilde{A}_{\ell} \tilde{j}_{\lambda}-\tilde{B}_{\ell} \tilde{y}_{\lambda}\right), \\
& \partial_{r} \tilde{B}_{\ell}=-\tilde{j}_{\lambda} V\left(\tilde{A}_{\ell} \tilde{j}_{\lambda}-\tilde{B}_{\ell} \tilde{y}_{\lambda}\right)
\end{aligned}
$$

with the boundary conditions [that follow from (24)]

$$
\tilde{A}_{\ell}(E, 0)=1, \quad \tilde{B}_{\ell}(E, 0)=0 .
$$

For any finite $r$, all the coefficient functions in Eqs. (43, 44) are entire functions of the parameter $E$ and the boundary conditions are $E$-independent. According to the Poincaré theorem [26] the solutions of these equations, i.e. the functions $\tilde{A}_{\ell}(E, r)$ and $\tilde{B}_{\ell}(E, r)$, are entire (analytic single-valued) functions of the complex variable $E$.

Therefore the structure we wanted to find is as follows:

$$
\begin{aligned}
& A_{\ell}(E, r)=\tilde{A}_{\ell}(E, r)+k^{2 \lambda+1} h(k) \tilde{B}_{\ell}(E, r), \\
& B_{\ell}(E, r)=k^{2 \lambda+1} \tilde{B}_{\ell}(E, r),
\end{aligned}
$$

where $\tilde{A}_{\ell}(E, r)$ and $\tilde{B}_{\ell}(E, r)$ are single-valued analytic functions of $E$. Apart from these single-valued functions, the original functions $A_{\ell}$ and $B_{\ell}$ involve the factors $k^{2 \lambda+1}$ and $h(k)$. Since $\lambda$ is half-integer, the power $(2 \lambda+1)$ is always even and thus $k^{2 \lambda+1}$ is also single-valued function of $E$, but $h(k)$ has a logarithmic branching point at $E=0$.

The functions $F_{\ell}^{(\text {in/out })}$ have similar structure

$$
\begin{aligned}
F_{\ell}^{(\text {in })}(E, r) & =\frac{1}{2}\left(A_{\ell}-i B_{\ell}\right)=\frac{1}{2}\left\{\tilde{A}_{\ell}(E, r)+k^{2 \lambda+1}[h(k)-i] \tilde{B}_{\ell}(E, r)\right\}, \\
F_{\ell}^{(\text {out })}(E, r)=\frac{1}{2}\left(A_{\ell}+i B_{\ell}\right) & =\frac{1}{2}\left\{\tilde{A}_{\ell}(E, r)+k^{2 \lambda+1}[h(k)+i] \tilde{B}_{\ell}(E, r)\right\} .
\end{aligned}
$$

\section{Analytic structure of the Jost functions}

What we have established in the previous Section, is valid for any complex $E$ and any finite distance $r$. In other words, so far we have established that the Jost functions (11) have the structure (48, 49) if the potential is cut off at certain radius $r=R$ (does not matter how 
large $R$ is). The problem is that to prove analyticity of $\tilde{A}_{\ell}(E, r)$ and $\tilde{B}_{\ell}(E, r)$ with respect to variable $E$, we used the Poincaré theorem which requires that the coefficients of Eqs. (43,44) be holomorphic functions of $E$. If $E$ is a real positive number and the potential is of a short range, this is true even if $r \rightarrow \infty$. Indeed, in such a case both $\tilde{j}_{\lambda}(k r)$ and $\tilde{y}_{\lambda}(k r)$ oscillate with finite amplitudes even at infinity and thus the coefficients of Eqs. (43,44) simply tend to zero (i.e. remain holomorphic) when $r \rightarrow \infty$. If however $E$ is negative or complex, then generally speaking this is not true. As we will see shortly, there still is a domain of complex $E$ where the coefficients remain holomorphic. In other words, if we extend $r$ to infinity, we have to narrow the domain of $E$.

The Riccati-Bessel and Riccati-Neumann functions are linear combinations of the RiccatHankel functions and thus at large distances behave as exponential functions (7). If the momentum has a nonzero imaginary part, then one or the other of these exponentials is diverging and thus both $\tilde{j}_{\lambda}(k r)$ and $\tilde{y}_{\lambda}(k r)$ tend to infinity when $r \rightarrow \infty$. To some extent the situation can be saved by using a short-range (exponentially decaying) potential $V(r) \sim \exp (-\eta r)$, which compensates the divergence of $\tilde{j}_{\lambda}(k r)$ and $\tilde{y}_{\lambda}(k r)$ within certain domain $\mathcal{D}$ of the complex $E$-plane along its real axis. The borders of the domain $\mathcal{D}$ are determined by the requirement that none of the coefficients of Eqs. (43]44) are divergent. The behavior (convergent or divergent) of these coefficients is determined by the product $\exp ( \pm 2 i k r) \exp (-\eta r)$. For a given $\eta$ it is not difficult to find the domain $\mathcal{D}$,

$$
\mathcal{D}=\left\{E:\left|2 \operatorname{Im} \sqrt{2 \mu E / \hbar^{2}}\right|<\eta\right\}
$$

which gives the condition

$$
(\operatorname{Im} E)^{2}<\frac{\hbar^{4} \eta^{4}}{16 \mu^{2}}+\frac{\hbar^{2} \eta^{2}}{2 \mu} \operatorname{Re} E .
$$

Similar analyticity domain was obtained by Motovilov [27] for the three-dimensional multichannel $T$-matrix, using a rigorous analysis of the corresponding scattering operators.

The faster the potential decays, the wider is the domain. An example of such a domain is shown in figure 2 for the model used in section 9 . It is a parabolic domain along the real axis, whose border is shown by the solid curve. It crosses the real axis at $E \approx-0.0332$ (in donor Hartree units). When $r \rightarrow \infty$, we can only use the Poincaré theorem within $\mathcal{D}$, and thus we can say that at least within this domain the functions $\tilde{A}_{\ell}(E, \infty)$ and $\tilde{B}_{\ell}(E, \infty)$ are the holomorphic functions of variable $E$.

If the potential $V(r)$ (or at least its long-range tail) is an analytic function of complex variable $r$ and exponentially decays along any ray $r=|r| e^{i \theta}$ within certain sector of the complex $r$ - 
plane, then the domain $\mathcal{D}$ can be extended by using the complex rotation described in section 4. With a complex radius, the product $\exp ( \pm 2 i k r) \exp (-\eta r)$ vanishes at infinity if $E$ is within

$$
\mathcal{D}=\{E:|2 \operatorname{Im}(k r)|<\eta \operatorname{Re} r\}
$$

which generalizes Eq. (50). It is easy to show that if $E=|E| \exp (i \chi)$ then such a domain can be defined by the following inequality

$$
\sin ^{2}\left(\frac{\chi}{2}+\theta\right)<\frac{\hbar^{2} \eta^{2} \cos ^{2} \theta}{8 \mu|E|} .
$$

With $\theta=0$ this condition is transformed into (151). An example of such a domain with the rotation angle $\theta=0.05 \pi$ for the potential (84) is shown by the dashed curve in Fig. 2 .

The physically interesting domain of the $E$-plane where the structure (48, 49), can be used in practical calculations, lies on the positive real axis (scattering) and in the close vicinity below it (pronounced resonances). Therefore, we can say that to all practical purposes this structure is valid at an arbitrary point $E$.

If we denote the asymptotic values (within $\mathcal{D}$ ) of the "tilded" functions as

$$
\tilde{a}_{\ell}(E)=\lim _{r \rightarrow \infty} \tilde{A}_{\ell}(E, r), \quad \tilde{b}_{\ell}(E)=\lim _{r \rightarrow \infty} \tilde{B}_{\ell}(E, r)
$$

Then the Jost functions and the $S$-matrix can be written as follows

$$
\begin{aligned}
f_{\ell}^{(\text {in })}(E) & =\frac{1}{2}\left\{\tilde{a}_{\ell}(E)+k^{2 \lambda+1}[h(k)-i] \tilde{b}_{\ell}(E)\right\}, \\
f_{\ell}^{(\text {out })}(E) & =\frac{1}{2}\left\{\tilde{a}_{\ell}(E)+k^{2 \lambda+1}[h(k)+i] \tilde{b}_{\ell}(E)\right\}, \\
s_{\ell}(E) & =\frac{\tilde{a}_{\ell}(E)+k^{2 \lambda+1}[h(k)+i] \tilde{b}_{\ell}(E)}{\tilde{a}_{\ell}(E)+k^{2 \lambda+1}[h(k)-i] \tilde{b}_{\ell}(E)} .
\end{aligned}
$$

To find the Jost functions or the $S$-matrix on any sheet of the Riemann surface, we need to calculate the functions $\tilde{a}_{\ell}(E)$ and $\tilde{b}_{\ell}(E)$ only once (because they are single valued). The choice of the sheet is determined by an appropriate choice of the value of the logarithmic function $h(k)$. Please note that $k^{2 \lambda+1}=k^{2 \ell}$ is a single-valued function of $E$. 


\section{Power-series expansions of the Jost functions}

The functions $\tilde{a}_{\ell}(E)$ and $\tilde{b}_{\ell}(E)$ are holomorphic (i.e. single-valued and analytic) and therefore can be expanded in Taylor series near any point $E_{0}$ within the domain $\mathcal{D}$ of the complex energy plane. The expansion around the point $E_{0}=0$ will give us the standard effective-range series. But we can also do such an expansion near an arbitrary point,

$$
\begin{aligned}
& \tilde{a}_{\ell}(E)=\sum_{n=0}^{\infty} \alpha_{n}^{(\ell)}\left(E_{0}\right)\left(E-E_{0}\right)^{n}, \\
& \tilde{b}_{\ell}(E)=\sum_{n=0}^{\infty} \beta_{n}^{(\ell)}\left(E_{0}\right)\left(E-E_{0}\right)^{n} .
\end{aligned}
$$

How the expansion coefficients $\alpha_{n}^{(\ell)}$ and $\beta_{n}^{(\ell)}$ can be found? For this purpose, we can derive differential equations, the solutions of which asymptotically tend to $\alpha_{n}^{(\ell)}$ and $\beta_{n}^{(\ell)}$. Indeed, such an expansion can be done at any fixed radius $r$ because the functions $\tilde{A}_{\ell}(E, r)$ and $\tilde{B}_{\ell}(E, r)$ reach their limits (54) at $r$ if the potential is cut off at this radius (in the spirit of the variable-phase approach). Therefore for each $r$, we have

$$
\begin{aligned}
& \tilde{A}_{\ell}(E, r)=\sum_{n=0}^{\infty} \mathcal{A}_{n}^{(\ell)}\left(E_{0}, r\right)\left(E-E_{0}\right)^{n}, \\
& \tilde{B}_{\ell}(E, r)=\sum_{n=0}^{\infty} \mathcal{B}_{n}^{(\ell)}\left(E_{0}, r\right)\left(E-E_{0}\right)^{n}
\end{aligned}
$$

where

$$
\alpha_{n}^{(\ell)}\left(E_{0}\right)=\lim _{r \rightarrow \infty} \mathcal{A}_{n}^{(\ell)}\left(E_{0}, r\right), \quad \beta_{n}^{(\ell)}\left(E_{0}\right)=\lim _{r \rightarrow \infty} \mathcal{B}_{n}^{(\ell)}\left(E_{0}, r\right) .
$$

Therefore, the differential equations mentioned above, should determine the functions $\mathcal{A}_{n}^{(\ell)}\left(E_{0}, r\right)$ and $\mathcal{B}_{n}^{(\ell)}\left(E_{0}, r\right)$. In order to obtain such equations, we expand the "tilded" functions $\tilde{j}_{\lambda}(E, r)$ and $\tilde{y}_{\lambda}(E, r)$ in the Taylor series near an arbitary point $E_{0}$

$$
\begin{aligned}
& \tilde{j}_{\lambda}(E, r)=\sum_{n=0}^{\infty} s_{n}^{(\lambda)}\left(E_{0}, r\right)\left(E-E_{0}\right)^{n}, \\
& \tilde{y}_{\lambda}(E, r)=\sum_{n=0}^{\infty} c_{n}^{(\lambda)}\left(E_{0}, r\right)\left(E-E_{0}\right)^{n},
\end{aligned}
$$


which are more general expansions than the series (35136) for the particular case of the threshold energy $E_{0}=0$. Any number of the expansion coefficients $s_{n}^{(\lambda)}\left(E_{0}, r\right)$ and $c_{n}^{(\lambda)}\left(E_{0}, r\right)$ can be found using the recurrence relations derived in the Appendix $B$.

Substituting the expansions (60,61,63,64) into Eqs. (43,44), and equalizing the factors of the same powers of $\left(E-E_{0}\right)$, we obtain the equations we are looking for,

$$
\begin{aligned}
& \partial_{r} \tilde{\mathcal{A}}_{n}^{(\ell)}=-\sum_{i+j+k=n} c_{i}^{(\lambda)} V\left(\tilde{\mathcal{A}}_{j}^{(\ell)} s_{k}^{(\lambda)}-\tilde{\mathcal{B}}_{j}^{(\ell)} c_{k}^{(\lambda)}\right), \\
& \partial_{r} \tilde{\mathcal{B}}_{n}^{(\ell)}=-\sum_{i+j+k=n} s_{i}^{(\lambda)} V\left(\tilde{\mathcal{A}}_{j}^{(\ell)} s_{k}^{(\lambda)}-\tilde{\mathcal{B}}_{j}^{(\ell)} c_{k}^{(\lambda)}\right),
\end{aligned}
$$

with the boundary conditions

$$
\tilde{\mathcal{A}}_{n}^{(\ell)}\left(E_{0}, 0\right)=\delta_{n 0}, \quad \tilde{\mathcal{B}}_{n}^{(\ell)}\left(E_{0}, 0\right)=0, \quad n=0,1,2,3, \ldots
$$

These conditions follow from the fact that the corresponding boundary conditions (45) do not depend on $E$. Therefore, starting with the initial values (67) at $r=0$, and numerically solving first $N+1$ pairs of differential equations of the system (65, 66) up to a sufficiently large radius $r_{\text {max }}$, we obtain first $N+1$ expansion coefficients

$$
\alpha_{n}^{(\ell)}\left(E_{0}\right)=\tilde{\mathcal{A}}_{n}^{(\ell)}\left(E_{0}, r_{\max }\right), \quad \beta_{n}^{(\ell)}\left(E_{0}\right)=\tilde{\mathcal{B}}_{n}^{(\ell)}\left(E_{0}, r_{\max }\right), \quad n=0,1,2, \ldots, N
$$

These coefficients give us the following approximate formulae for the Jost functions

$$
\begin{aligned}
f_{\ell}^{(\text {in })}(E) & \approx \frac{1}{2} \sum_{n=0}^{N}\left\{\alpha_{n}^{(\ell)}\left(E_{0}\right)+k^{2 \lambda+1}[h(k)-i] \beta_{n}^{(\ell)}\left(E_{0}\right)\right\}\left(E-E_{0}\right)^{n}, \\
f_{\ell}^{(\text {out })}(E) & \approx \frac{1}{2} \sum_{n=0}^{N}\left\{\alpha_{n}^{(\ell)}\left(E_{0}\right)+k^{2 \lambda+1}[h(k)+i] \beta_{n}^{(\ell)}\left(E_{0}\right)\right\}\left(E-E_{0}\right)^{n},
\end{aligned}
$$

which are valid for any complex value of $E$ within a domain around the chosen central point $E_{0}$. Apparently, the closer $E$ is to $E_{0}$, the better is the accuracy of these formulae. It is interesting to note that Eq. (9) of Ref. [10] is the first term of our Eq. (69) for the particular case of $E_{0}=0$.

An alternative way of using formulae (6970) is to treat the expansion coefficients $\alpha_{n}^{(\ell)}\left(E_{0}\right)$, $\beta_{n}^{(\ell)}\left(E_{0}\right), n=0,1, \ldots, N$ as fitting parameters. Adjusting them in such a way that the 
corresponding cross section (see Appendix A.4) reproduces experimental data in vicinity of a real energy $E_{0}$, one then can use the Jost function (69) at the nearby complex energies for locating possible resonances. The obvious advantage of such an approach is that the resonance energy and the width are deduced directly from experimental data using correct analytic structure of the $S$-matrix.

\section{Effective-range expansion}

Far away from the interaction region the radial wave function (18) is a linear combination of the Riccati-Bessel and Riccati-Neumann functions

$$
u_{\ell}(E, r) \underset{r \rightarrow \infty}{\longrightarrow} a_{\ell}(E) j_{\lambda}(k r)-b_{\ell}(E) y_{\lambda}(k r)
$$

where

$$
a_{\ell}(E)=\lim _{r \rightarrow \infty} A_{\ell}(E, r), \quad b_{\ell}(E)=\lim _{r \rightarrow \infty} B_{\ell}(E, r) .
$$

The functions $j_{\lambda}$ and $y_{\lambda}$ in (71) can be written in their asymptotic form,

$$
\begin{aligned}
& j_{\lambda}(k r) \underset{r \rightarrow \infty}{\longrightarrow} \sin \left(k r-\frac{\lambda \pi}{2}\right), \\
& y_{\lambda}(k r) \underset{r \rightarrow \infty}{\longrightarrow}-\cos \left(k r-\frac{\lambda \pi}{2}\right),
\end{aligned}
$$

which gives

$$
\begin{array}{cl}
u_{\ell}(E, r) \underset{r \rightarrow \infty}{\longrightarrow} \quad a_{\ell}(E) \sin \left(k r-\frac{\lambda \pi}{2}\right)+b_{\ell}(E) \cos \left(k r-\frac{\lambda \pi}{2}\right)= \\
=N \sin \left[k r-\frac{\lambda \pi}{2}+\delta_{\ell}(E)\right],
\end{array}
$$

where $a_{\ell}$ and $b_{\ell}$ are replaced with their common normalization factor $N$ and the scattering phase shift $\delta_{\ell}$,

$$
\begin{aligned}
& a_{\ell}(E)=N \cos \delta_{\ell}(E) \\
& b_{\ell}(E)=N \sin \delta_{\ell}(E) .
\end{aligned}
$$


Using the relations (46, 47) at large distances $(r \rightarrow \infty)$,

$$
\begin{aligned}
a_{\ell}(E) & =\tilde{a}_{\ell}(E)+k^{2 \lambda+1} h(k) \tilde{b}_{\ell}(E), \\
b_{\ell}(E) & =k^{2 \lambda+1} \tilde{b}_{\ell}(E),
\end{aligned}
$$

we can construct the so called effective-range function which is a holomorphic function of the energy. This is done by taking the ratio

$$
\cot \delta_{\ell}=\frac{a_{\ell}}{b_{\ell}}=\frac{\tilde{a}_{\ell}+k^{2 \lambda+1} h \tilde{b}_{\ell}}{k^{2 \lambda+1} \tilde{b}_{\ell}}
$$

and moving all the "troublesome" terms and factors which may generate singularities, to the left hand side of the equation,

$$
\begin{gathered}
k^{2 \lambda+1} \cot \delta_{\ell}=\frac{\tilde{a}_{\ell}}{\tilde{b}_{\ell}}+k^{2 \lambda+1} h \\
k^{2 \lambda+1}\left[\cot \delta_{\ell}(E)-h(k)\right]=k^{2 \ell}\left[\cot \delta_{\ell}(E)-h(k)\right]=\frac{\tilde{a}_{\ell}(E)}{\tilde{b}_{\ell}(E)} .
\end{gathered}
$$

Both the numerator and denominator in the last ratio can be written in the form of power series (58, 59) with $E_{0}=0$

$$
k^{2 \lambda+1}\left[\cot \delta_{\ell}(E)-h(k)\right]=\frac{\sum_{n=0}^{\infty} \alpha_{n}^{(\ell)}(0) E^{n}}{\sum_{n=0}^{\infty} \beta_{n}^{(\ell)}(0) E^{n}} .
$$

Using Eq. (3.6.22) of the book by Abramowitz et al.,

$\frac{a_{0}+a_{1} x+a_{2} x^{2}+\cdots}{b_{0}+b_{1} x+b_{2} x^{2}+\cdots}=\frac{a_{0}}{b_{0}}\left[1+\left(\frac{a_{1}}{a_{0}}-\frac{b_{1}}{b_{0}}\right) x+\left(\frac{a_{2}}{a_{0}}-\frac{b_{1}\left(a_{1} b_{0}-a_{0} b_{1}\right)}{a_{0} b_{0}^{2}}-\frac{b_{2}}{b_{0}}\right) x^{2}+\cdots\right]$, the division of two polynomials in Eq. (

$$
k^{2 \lambda+1}\left[\cot \delta_{\ell}(E)-h(k)\right]=-\frac{1}{a^{(\ell)}}+\frac{r_{0}^{(\ell)}}{2} k^{2}+\cdots,
$$


where the scattering length $a^{(\ell)}$ and the effective radius $r_{0}^{(\ell)}$ for the state with the angular momentum $\ell$ are given by

$$
\begin{aligned}
a^{(\ell)} & =-\frac{\beta_{0}^{(\ell)}}{\alpha_{0}^{(\ell)}} \\
r_{0}^{(\ell)} & =\frac{\hbar^{2}}{\mu}\left(\frac{\alpha_{1}^{(\ell)}}{\beta_{0}^{(\ell)}}-\frac{\alpha_{0}^{(\ell)} \beta_{1}^{(\ell)}}{\beta_{0}^{(\ell) 2}}\right) .
\end{aligned}
$$

\section{A numerical example related to quantum dot the- ory}

To demonstarate how the proposed method works, we use the following circularly-symmetric potential, which is motivated by the models that are currently used in the theory of quantum dots,

$$
U(r)=V_{0}\left(r-r_{0}\right) e^{-r / R},
$$

with $V_{0}=25, r_{0}=2$, and $R=2$, where $V_{0}$ (as well as all the energies in this example) is measured in the so called "donor Hartree units" and the distances in the units of "donor Bohr radius", which were chosen to be $10.96 \mathrm{meV}$ and $101.89 \AA$, respectively. These values for the units are relevant to the motion of electrons in the semiconductor material GaAs [28], where the effective electron mass is $\mu=0.063 m_{e}$ (with $m_{e}$ being free electron mass).

Although, strictly, the potential (84) should be considered as an abstract quantum-mechanical "toy" model, we chose its shape in such a way that it resembles the potentials that are currently used to describe two-dimensional quantum dots (see, for example, Refs. [28--31]). As is seen in Fig, 4 , our potential has a repulsive barrier which is not present in the traditional quantum-dot models. The main reason for introducing such a barrier was to enrich our "toy" model spectrum with resonances. However, one can argue that such a barrier may appear in real quantum dots as well. Indeed, when electrons fill up the lower levels of a dot, they should repel each other and tend to stay mostly at its periphery. This means that for an additional incoming electron the attractive force at the centre is reduced and a repulsion appears at the border. In other words, the original empty-dot confining-potential (shown with the dashed curve) is transformed into something that looks like our "toy" potential. Of course, this speculative reasoning does not mean that we claim that our potential is anything more than an abstract model. 
Since nothing special is associated with the angular momentum, we only consider here the $S$-wave states $(\ell=0)$. For such a case, the potential (84) supports three bound states and a squence of resonances. These spectral points (given in Table 1 and shown in Fig. 3) were located using the exact approach, i.e. as the roots of Eq. (9), where $f_{\ell}^{(\mathrm{in})}$ is the asymptotic value (11) of the solution of Eq. (14).

To make sure that we did not miss any of the bound states and/or narrow resonances, we calculated the $S$-wave scattering phase-shift and checked if it obeys the Levinson's theorem. In Refs. [9, 32, 33] it was shown that in the absence of a zero-energy bound state for the $P$-wave and always for the $S$-wave, this theorem is the same as for the three-dimensional scattering, namely,

$$
\delta_{\ell}(0)-\delta_{\ell}(\infty)=\pi N_{\ell},
$$

where $N_{\ell}$ is the number of bound states with the angular momentum $\ell$. If the energy moves to the right along the real axis, the phase shift increases by $\pi$ near each resonance which is not far from the real axis. The smaller is the width, the more sharp is the increase. When calculating the phase shift numerically, it is easy to miss a sharp jump corresponding a narrow resonance. The curve "A" in Fig. [5 is an example of such omissions (the first two resonances are missed because of a too large step along the $E$-axis). The correct phase shift is shown by the curve "B". It starts with $3 \pi$ at the threshold and tends to zero at the infinity, in accordance with Eq. (85).

Calculating the first two expansion coefficients and using Eqs. (82,83), we found the following scattering length and effective radius,

$$
a_{0}=-0.4521260323[\text { dimensionless }], \quad r_{0}=0.0586790752\left[\text { length }^{2}\right] .
$$

As a first test of the expansions (69/70), we performed them at several scattering energies (i.e. on the real energy axis) and compared the approximate cross section obtained from the approximate Jost functions (see Appendix A.4) with the corresponding exact cross section that was calculated using the exact Jost functions via numerical integration of the system of differential equations (14]15). Fig. 6 shows the exact cross section in the interval $E \in(0,10]$ (thick curve) and the approximate cross sections (thin curves) when only the first five terms of the series (69) were taken into account for $E_{0}$ being 1, 5, and 7. It is seen that within rather wide interval around each $E_{0}$ the expansion reproduces the cross section very well even with all its zigzags.

The next step was to test our expansions at complex energies. To begin with, we performed them around a point on the real axis, namely, around $E_{0}=7$ (far away from the threshold 
energy) and looked at the Jost function at the nearby complex energies. Why 7 ? Simply because there is a resonance not far from this point (third resonance of Table 1). To check the accuracy of the expansion, we compared the approximate values of $f_{\ell}^{(\mathrm{in})}(E)$ at various points around $E_{0}$ with the corresponding exact values of the Jost function. Apparently, the closer the point $E$ is to the center of the expansion, the more accurate should be the result. Fig. 7 shows three closed contours around $E_{0}=7$. Within the smallest of them the relative error of $f_{\ell}^{(\mathrm{in})}(E)$ obtained by the expansion (69) with $N=4$ is less than $1 \%$. The other two contours show the domains of $5 \%$ and $10 \%$ accuracy. The important fact is that even if the expansion is done on the real axis, the semi-analytic formulae (69070) remain valid at the nearby complex points.

The star in Fig. 7 is a resonant zero of the exact Jost function. As is seen, the $1 \%$-contour has a "dent" near this point. The reason for it is that in calculating the relative error, we have an exact value of $f_{\ell}^{(\text {in) }}(E)$ in the denominator and this value is zero at the resonance. By the way, the approximate Jost function (69) with $N=4$ has zero at $E=7.1051679246-\frac{i}{2} 0.5683685515$ which is not far from its exact position. This means that the expansion done on the real axis can be used for locating narrow resonances.

Finally, we tested the expansion around a point in the fourth quadrant (where the resonances are) of the complex energy plane. When solving the differential equations (65,65) we used the complex rotation of the coordinate (see $\operatorname{Sec} 4$ ) with such an angle $\theta$ that $\operatorname{Im}\left(k_{0} r\right)=0$ (where $k_{0}$ is the momentum corresponding to $\left.E_{0}\right)$. This guarantees that $E_{0}$ is within the domain $\mathcal{D}$ (see Sec,6).

Fig. 8 shows the exact positions of two resonances (indicated with stars), the center of the expansion (cross) at $E_{0}=7.55-i 1.06$ which is in the middle between them, and two pairs of the approximate locations of these resonances: open circles for three terms of the expansion and filled circles for five expansion terms. It is seen that the expansion converges, i.e. the more terms are taken into account, the more accurately the resonances are reproduced. It should be noted that the chosen position of $E_{0}$ is the "worst case". If we move $E_{0}$ a bit closer towards one of the resonances, it is reproduced much more accurately.

\section{Conclusion}

In this paper, we show that the Jost function for the two-dimension scattering can be written as a sum of two terms, one of which is an analytic single-valued function of the energy $E$ 
while the other term can be factorized in an analytic function of $E$ and a logarithmic function of the momentum. This means that the (logarithmic) branching point of the Riemann energy-surface is given in the Jost function explicitly via the logarithmic factor. The remaining energy-dependent functions are defined on single energy plane which does not have any branching points anymore. For these energy-dependent functions, we derive a system of firstorder differential equations. Then, using the fact that the functions are analytic within certain domain $\mathcal{D}$, we expand them in the power series around an arbitrary point $E_{0} \in \mathcal{D}$ and obtain a system of differential equations that determine the expansion coefficients.

A systematic procedure developed in this paper, allows us to accurately calculate the power series expansion of the Jost function practically at any point on the Riemann surface of the energy. Actually, the expansion is done for the single-valued functions of the energy, while the choice of the sheet of the Riemann surface is done by appropriately choosing the sheet of the logarithmic function of the momentum.

The method suggested in this paper, makes it possible to obtain a semi-analytic expression for the two-dimensional Jost function (and therefore for the corresponding $S$-matrix) near an arbitrary point on the Riemann surface and thus to locate the resonant states as the $S$-matrix poles. Alternatively, the expansion can be used to parametrize experimental data, where the unknown expansion coefficients are the fitting parameters. Such a parametrization will have the correct analytic structure. After fitting the data given at real energies, one can use the semianalytic Jost function to search for resonances in the nearby domain of the Riemann surface. The efficiency and accuracy of the suggested expansion is demonstrated by an example of a two-dimensional model potential.

\section{APPENDICES}

\section{A Two-dimensional partial-wave decomposition}

The partial-wave decomposition of the wave function, scattering amplitude, and cross section for a particle moving on a plane, is done using the cylindrical coordinates where the $z$-axis (perpendicular to the plane) is needed to define the orbital angular momentum. All the steps of such a decomposition are similar to the three-dimensional case, but the resulting formulae are not obvious and cannot be easily obtained from the corresponding $3 D$-analysis. The derivations of various formulae of this type are given in several different papers (see, for example, Refs. [34,35]). Usually these derivations are very concise with many details omitted. Since such derivations are not present in the standard textbooks on quantum mechanics, we 
feel that it is worthwhile to collect everything in one place. This is why we include this Appendix.

\section{A.1 Radial Schrödinger equation}

Consider a particle of mass $\mu$, moving on a plane and being affected by a force that is described by a potential $U(\vec{r})$, which is assumed to be of a short-range and circularly symmetric,

$$
U(\vec{r})=U(|\vec{r}|)
$$

To make the derivations simple, we assume that our particle does not have spin (this restriction can be easily revoked later). In the coordinate representation, the Hamiltonian $H$ of such a particle is most conveniently expressed using the polar coordinates,

$$
\begin{aligned}
H & =-\frac{\hbar^{2}}{2 \mu} \Delta+U \\
\Delta & =\frac{1}{r} \frac{\partial}{\partial r}\left(r \frac{\partial}{\partial r}\right)+\frac{1}{\hbar^{2} r^{2}} \hat{L}^{2},
\end{aligned}
$$

where

$$
\hat{L}^{2}=\hbar^{2} \frac{\partial^{2}}{\partial \varphi^{2}}
$$

is the two-dimensional operator of the square of the angular momentum. Its eigenfunctions $\mathcal{Y}_{m}(\varphi)$ obeying the equation

$$
\hat{L}^{2} \mathcal{Y}_{m}(\varphi)=-\hbar^{2} m^{2} \mathcal{Y}_{m}(\varphi)
$$

and normalized as

$$
\int_{0}^{2 \pi} \mathcal{Y}_{m}^{*}(\varphi) \mathcal{Y}_{m^{\prime}}(\varphi) d \varphi=\delta_{m m^{\prime}}
$$

are easy to find,

$$
\mathcal{Y}_{m}(\varphi)=\frac{1}{\sqrt{2 \pi}} e^{i m \varphi}, \quad m=0, \pm 1, \pm 2, \ldots
$$

From the definition of the Fourier series on the interval $[0,2 \pi]$ it follows that

$$
\sum_{m=-\infty}^{+\infty} \mathcal{Y}_{m}(\varphi) \mathcal{Y}_{m}^{*}\left(\varphi^{\prime}\right)=\delta\left(\varphi-\varphi^{\prime}\right)
$$


Each value of the angular momentum (except for zero) is represented twice: with two opposite signs. Classically, these two states correspond to the motion of the particle at the same distance $r$ from the center and with the same velocity, but at different sides of the center (see Fig. 9).

The quantum number of the angular momentum $\ell=|m|$ is always non-negative and irrespective of its magnitude (if $\ell \neq 0$ ) the vector $\vec{\ell}$ can have two (only two) directions: up or down (like the spin 1/2). The quantum number $m= \pm \ell$ is its $z$-component. In principle, we can use the same notation for the eigenfunctions of the operator $\hat{L}^{2}$ as in the three-dimensional case, namely, $\mathcal{Y}_{\ell m}$ with two subscripts. However, because of the relation $m= \pm \ell$, the subscript $\ell$ is redundant.

This can be formulated in a different way. The functions (87) form a complete ortho-normal set on the interval $\varphi \in[0,2 \pi]$. This means that any (reasonable) function $f(\varphi)$ defined on this interval, can be written as their linear combination, and such a combination can be written in the following two (equivalent) ways

$$
f(\varphi)=\sum_{m=-\infty}^{+\infty} a_{m} \mathcal{Y}_{m}(\varphi)=\sum_{\ell=0}^{\infty} \sum_{m= \pm \ell} \tilde{a}_{\ell m} \mathcal{Y}_{m}(\varphi), \quad a_{m}=\tilde{a}_{|m| m}
$$

In other words the following summations are equivalent

$$
\sum_{m=-\infty}^{+\infty} \longleftrightarrow \sum_{\ell=0}^{\infty} \sum_{m= \pm \ell}
$$

The operator corresponding to the quantum number $m$ is obtained as follows. The gradient operator in the cylindrical coordinates is

$$
\vec{\nabla}=\hat{\vec{r}} \frac{\partial}{\partial r}+\hat{\vec{\varphi}} \frac{1}{r} \frac{\partial}{\partial \varphi}+\hat{\vec{z}} \frac{\partial}{\partial z}
$$

where

$$
\hat{\vec{r}}=[\hat{\vec{\varphi}} \times \hat{\vec{z}}], \quad \hat{\vec{\varphi}}=[\hat{\vec{z}} \times \hat{\vec{r}}], \quad \hat{\vec{z}}=[\hat{\vec{r}} \times \hat{\vec{\varphi}}]
$$

are the corresponding unit vectors. Then

$$
[\vec{r} \times \vec{p}]=r \hat{\vec{r}} \times \frac{\hbar}{i} \vec{\nabla}=\frac{\hbar r}{i}\left(\hat{\vec{z}} \frac{1}{r} \frac{\partial}{\partial \varphi}-\hat{\vec{\varphi}} \frac{\partial}{\partial z}\right)
$$

and thus

$$
\ell_{z}=\frac{\hbar}{i} \frac{\partial}{\partial \varphi}
$$


Apparently, both $\hat{L}^{2}$ and $\ell_{z}$ commute with the Hamiltonian. The quantum numbers $\ell$ and $m$ are therefore conserving. When specifying $m$, we implicitly specify the quantum number $\ell$ as well. This means that the quantum state of the particle is determined by two conserving quantum numbers, namely, the energy $E$ and the $z$-component of the angular momentum $m$. The corresponding wave function, obeying the Schrödinger equation,

$$
H \psi_{E m}(\vec{r})=E \psi_{E m}(\vec{r}),
$$

can be factorized in the radial and angular parts

$$
\psi_{E m}(\vec{r})=\frac{u_{m}(E, r)}{\sqrt{r}} \mathcal{Y}_{m}(\varphi),
$$

where $\sqrt{r}$ in the denominator is introduced to obtain the radial equation without the first derivative. Substituting this factorized form into the Schrödinger equation, we obtain

$$
\left[\frac{d^{2}}{d r^{2}}+k^{2}-\frac{m^{2}-1 / 4}{r^{2}}-V(r)\right] u_{m}(E, r)=0,
$$

where $k$ is the wave number (linear momentum) defined by

$$
k^{2}=\frac{2 \mu}{\hbar^{2}} E
$$

and $V(r)$ is the reduced (in the units of $[\text { length }]^{-2}$ ) potential

$$
V(r)=\frac{2 \mu}{\hbar^{2}} U(r) .
$$

Noting that Eq. (91) is exactly the same for both choices of the sign for $m$, we conclude that $u_{m}(E, r)$ actually depends on $\ell$ but not on $m$. The radial equation can therefore be re-written in the way we used to see it in the three-dimensional problems

$$
\left[\frac{d^{2}}{d r^{2}}+k^{2}-\frac{\lambda(\lambda+1)}{r^{2}}-V(r)\right] u_{\ell}(E, r)=0,
$$

where we introduced

$$
\lambda=\ell-\frac{1}{2}
$$

and did the replacement

$$
\ell^{2}-\frac{1}{4}=\left(\ell-\frac{1}{2}\right)\left(\ell+\frac{1}{2}\right)=\lambda(\lambda+1) .
$$


Formally, Eq. (93) looks exactly like the radial equation of the three-dimensional problem. The only difference is that $\lambda$ is not an integer number

$$
\lambda=-\frac{1}{2}, \frac{1}{2}, \frac{3}{2}, \frac{5}{2}, \ldots
$$

This simple fact makes a huge difference: it changes the analytic properties of the Jost function and thus the $S$-matrix, because the Riccat-Neumann function $y_{\lambda}(k r)$ with a half-integer $\lambda$ has a logarithmic branching point on the Riemann surface of the energy [17].

\section{A.2 Plane-wave and circular waves}

Consider a two-dimensional plane wave normalized to the $\delta$-function

$$
\langle\vec{r} \mid \vec{k}\rangle=\frac{e^{i \vec{k} \vec{r}}}{2 \pi}, \quad\left\langle\vec{k}^{\prime} \mid \vec{k}\right\rangle=\delta\left(\vec{k}^{\prime}-\vec{k}\right)=\frac{1}{k} \delta\left(k^{\prime}-k\right) \delta\left(\varphi^{\prime}-\varphi\right)
$$

where $\varphi$ is the polar angle of the momentum $\hbar \vec{k}$. This plane wave can be expanded over the full set $\{\mathcal{Y}\}$ of the angular functions (87),

$$
\frac{e^{i \vec{k} \vec{r}}}{2 \pi}=\frac{e^{i k r \cos \varphi}}{2 \pi}=\sum_{\ell m} a_{m}(k r) \mathcal{Y}_{m}(\varphi)
$$

where the $x$-axis is directed along the coordinate vector $\vec{r}$. The expansion coefficients

$$
a_{m}(k r)=\frac{1}{(2 \pi)^{3 / 2}} \int_{0}^{2 \pi} e^{i(k r \cos \varphi-m \varphi)} d \varphi
$$

can be found using the integral representation of the Bessel function [17]

$$
\begin{aligned}
J_{m}(z) & =\frac{1}{\pi i^{m}} \int_{0}^{\pi} e^{i z \cos \varphi} \cos (m \varphi) d \varphi=\frac{1}{2 \pi i^{m}} \int_{0}^{\pi} e^{i z \cos \varphi}\left(e^{i m \varphi}+e^{-i m \varphi}\right) d \varphi \\
& =\frac{1}{2 \pi i^{m}} \int_{-\pi}^{\pi} e^{i(z \cos \varphi-m \varphi)} d \varphi=\frac{i^{m}}{2 \pi} \int_{0}^{2 \pi} e^{i(-z \cos \varphi-m \varphi)} d \varphi
\end{aligned}
$$

Comparing Eq. (97) with (98) and using the symmetry property of the Bessel function $J_{m}(-z)=(-1)^{m} J_{m}(z)$, we see that

$$
a_{m}(k r)=\frac{i^{m}}{\sqrt{2 \pi}} J_{m}(k r)
$$


and thus

$$
\frac{e^{i \vec{k} \vec{r}}}{2 \pi}=\frac{1}{2 \pi} \sum_{\ell m} i^{m} e^{i m \varphi} J_{m}(k r)=\frac{1}{2 \pi} \sum_{-\infty}^{+\infty} i^{m} e^{i m \varphi} J_{m}(k r)=\frac{1}{\sqrt{2 \pi}} \sum_{-\infty}^{+\infty} i^{m} J_{m}(k r) \mathcal{Y}_{m}(\varphi)
$$

Using another symmetry property, $J_{-m}(z)=(-1)^{m} J_{m}(z)$, we see that the product $i^{m} J_{m}(k r)$ does not depend on the sign of $m$ and thus this expansion can be re-written as

$$
\begin{aligned}
\frac{e^{i \vec{k} \vec{r}}}{2 \pi} & =\frac{1}{2 \pi}\left[J_{0}(k r)+\sum_{\ell=1}^{\infty} i^{\ell}\left(e^{i \ell \varphi}+e^{-i \ell \varphi}\right) J_{\ell}(k r)\right] \\
& =\frac{1}{2 \pi} \sum_{\ell=0}^{\infty} \epsilon_{\ell} i^{\ell} \cos (\ell \varphi) J_{\ell}(k r)
\end{aligned}
$$

where $\epsilon_{\ell}$ is the "multiplicity" of an $\ell$-state, i.e. is the analog of the factor $(2 \ell+1)$ of the 3D-case,

$$
\epsilon_{\ell}= \begin{cases}1, & \ell=0 \\ 2, & \ell>0\end{cases}
$$

Expressing the Bessel function via the Riccati-Hankel functions,

$$
J_{\ell}(z)=\sqrt{\frac{1}{2 \pi z}}\left[h_{\ell-1 / 2}^{(-)}(z)+h_{\ell-1 / 2}^{(+)}(z)\right]
$$

we obtain the following decomposition of the $2 D$ plane wave in the incoming $(-)$ and outgoing $(+)$ circular waves

$$
\frac{e^{i \vec{k} \vec{r}}}{2 \pi}=\frac{1}{2 \pi \sqrt{k r}} \sum_{\ell=0}^{\infty} \sum_{m= \pm \ell} i^{\ell}\left[h_{\lambda}^{(-)}(k r)+h_{\lambda}^{(+)}(k r)\right] \mathcal{Y}_{m}(\varphi)
$$

where $\lambda$ is defined by Eq. (94).

In the above, we assumed that vector $\vec{r}$ was directed along the $x$-axis. If this is not the case, then the dot-product

$$
\vec{k} \vec{r}=k r\left(\cos \varphi_{k} \cos \varphi_{r}+\sin \varphi_{k} \sin \varphi_{r}\right)=k r \cos \left(\varphi_{r}-\varphi_{k}\right)
$$

depends on the two polar angles. In this general case the plane wave is expanded over two sets of functions $\left\{\mathcal{Y}\left(\varphi_{k}\right)\right\}$ and $\left\{\mathcal{Y}\left(\varphi_{r}\right)\right\}$ depending on the angles of the momentum and co-ordinate 
vectors. In a similar way as we did it above, it is not difficult to show that

$$
\begin{aligned}
\frac{e^{i \vec{k} \vec{r}}}{2 \pi} & =\sum_{-\infty}^{+\infty} i^{m} J_{m}(k r) \mathcal{Y}_{m}^{*}\left(\varphi_{r}\right) \mathcal{Y}_{m}\left(\varphi_{k}\right)=\sum_{\ell=0}^{\infty} i^{\ell} J_{\ell}(k r) \sum_{m= \pm \ell} \mathcal{Y}_{m}^{*}\left(\varphi_{r}\right) \mathcal{Y}_{m}\left(\varphi_{k}\right) \\
& =\frac{1}{2 \pi} \sum_{\ell=0}^{\infty} \epsilon_{\ell} i^{\ell} \cos \left[\ell\left(\varphi_{r}-\varphi_{k}\right)\right] J_{\ell}(k r) \\
& =\frac{1}{\sqrt{2 \pi k r}} \sum_{\ell m} i^{\ell}\left[h_{\lambda}^{(-)}(k r)+h_{\lambda}^{(+)}(k r)\right] \mathcal{Y}_{m}^{*}\left(\varphi_{r}\right) \mathcal{Y}_{m}\left(\varphi_{k}\right) \\
& =\frac{1}{\sqrt{2 \pi k r}} \sum_{\ell m} i^{\ell} u_{\ell}^{(0)}(E, r) \mathcal{Y}_{m}^{*}\left(\varphi_{r}\right) \mathcal{Y}_{m}\left(\varphi_{k}\right)
\end{aligned}
$$

where

$$
u_{\ell}^{(0)}(E, r)=h_{\lambda}^{(-)}(k r)+h_{\lambda}^{(+)}(k r)=2 j_{\lambda}(k r)
$$

is a regular solution of the radial Schrödinger equation (93) for the case $V(r) \equiv 0$. These partial-wave decompositions can be conveniently written in the following symbolic form

$$
\begin{gathered}
|\vec{k}\rangle=\sum_{\ell m}|k \ell m\rangle \mathcal{Y}_{m}\left(\varphi_{k}\right), \quad|\vec{r}\rangle=\sum_{\ell m}|r \ell m\rangle \mathcal{Y}_{m}\left(\varphi_{r}\right), \\
\left\langle r \ell m \mid k \ell^{\prime} m^{\prime}\right\rangle=\delta_{\ell \ell^{\prime}} \delta_{m m^{\prime}} i^{\ell} J_{\ell}(k r)=\delta_{\ell \ell^{\prime}} \delta_{m m^{\prime}} i^{\ell} \sqrt{\frac{2}{\pi k r}} j_{\lambda}(k r), \\
\langle\vec{r} \mid k \ell m\rangle=i^{\ell} \sqrt{\frac{2}{\pi k r}} j_{\lambda}(k r) \mathcal{Y}_{m}^{*}\left(\varphi_{r}\right), \quad\langle\vec{k} \mid r \ell m\rangle=(-i)^{\ell} \sqrt{\frac{2}{\pi k r}} j_{\lambda}(k r) \mathcal{Y}_{m}^{*}\left(\varphi_{k}\right), \\
\left\langle k \ell m \mid k^{\prime} \ell^{\prime} m^{\prime}\right\rangle=\frac{1}{k} \delta\left(k-k^{\prime}\right) \delta_{\ell \ell^{\prime}} \delta_{m m^{\prime}}, \quad\left\langle r \ell m \mid r^{\prime} \ell^{\prime} m^{\prime}\right\rangle=\frac{1}{r} \delta\left(r-r^{\prime}\right) \delta_{\ell \ell^{\prime}} \delta_{m m^{\prime}}, \\
\int_{0}^{\infty} \sum_{\ell m}|k \ell m\rangle\left\langle k \ell m\left|k d k=1, \quad \int_{0}^{\infty} \sum_{\ell m}\right| r \ell m\right\rangle\langle r \ell m| r d r=1 .
\end{gathered}
$$

\section{A.3 Scattering wave function}

The plane wave (104) is a scattering wave function $\psi_{\vec{k}}(\vec{r})$ for the particular case of $V(r) \equiv 0$. Apparently, the structure of its partial-wave decomposition should be the same for all potentials

$$
\psi_{\vec{k}}(\vec{r})=\frac{N}{\sqrt{2 \pi k r}} \sum_{\ell m} i^{\ell} u_{\ell}(E, r) \mathcal{Y}_{m}^{*}\left(\varphi_{r}\right) \mathcal{Y}_{m}\left(\varphi_{k}\right)
$$


where the factor $N$ is determined by the choice of the potential and the collision energy (for the free motion, $N=1$ at all energies). The purpose of this factor is to always have exactly the same normalization, namely,

$$
\left\langle\psi_{\vec{k}} \mid \psi_{\vec{k}^{\prime}}\right\rangle=\delta\left(\vec{k}-\vec{k}^{\prime}\right)
$$

An appropriate value for $N$ can be found as follows. The Riccati-Hankel functions $h_{\lambda}^{( \pm)}(k r)$ are two linearly independent solutions of the radial Schrödinger equation (93) without the potential term. This means that for a short-range potential, its solution asymptotically behaves as a linear combination of the Riccati-Hankel functions,

$$
u_{\ell}(E, r) \underset{r \rightarrow \infty}{\longrightarrow} f_{\ell}^{(\mathrm{in})}(E) h_{\lambda}^{(-)}(k r)+f_{\ell}^{(\mathrm{out})}(E) h_{\lambda}^{(+)}(k r)
$$

where the combination coefficients depend on the energy and are called the Jost functions. In fact, they are the amplitudes of the incoming $(-)$ and outgoing $(+)$ circular waves. On the other hand, at large distances the wave function $\psi_{\vec{k}}(\vec{r})$ consists of the two parts: the initial (incident) wave $\psi_{\vec{k}}^{(0)}(\vec{r})$ and a scattered circular wave that goes in all directions with certain amplitude $F$,

$$
\psi_{\vec{k}}(\vec{r}) \underset{r \rightarrow \infty}{\longrightarrow} \psi_{\vec{k}}^{(0)}(\vec{r})+F\left(E, \varphi_{k}, \varphi_{r}\right) \frac{e^{i k r}}{\sqrt{r}} .
$$

If $\psi_{\vec{k}}(\vec{r})$ is properly normalized then $\psi_{\vec{k}}^{(0)}(\vec{r}) \equiv e^{i \vec{k} \vec{r}} /(2 \pi)$. This means that the radial wave function at large distances should also be split in two parts one of which coincides with the function (105). In doing such a splitting of the function (112), we obtain

$$
u_{\ell}(E, r) \underset{r \rightarrow \infty}{\longrightarrow} f_{\ell}^{(\mathrm{in})}\left[h_{\lambda}^{(-)}+h_{\lambda}^{(+)}+\left(\frac{f_{\ell}^{(\text {out })}}{f_{\ell}^{(\text {in })}}-1\right) h_{\lambda}^{(+)}\right]
$$

and see that $N=1 / f_{\ell}^{(\text {in })}$, i.e.

$$
\psi_{\vec{k}}(\vec{r})=\frac{1}{\sqrt{2 \pi k r} f_{\ell}^{(\mathrm{in})}(E)} \sum_{\ell m} i^{\ell} u_{\ell}(E, r) \mathcal{Y}_{m}^{*}\left(\varphi_{r}\right) \mathcal{Y}_{m}\left(\varphi_{k}\right)
$$

\section{A.4 Cross section}

Defining the partial-wave $S$-matrix and the amplitude,

$$
s_{\ell}(E)=\frac{f_{\ell}^{(\text {out })}(E)}{f_{\ell}^{\text {(in) }}(E)}, \quad f_{\ell}(E)=\frac{s_{\ell}(E)-1}{\sqrt{2 \pi i k}}
$$


and using

$$
h_{\lambda}^{(+)}(k r) \underset{r \rightarrow \infty}{\longrightarrow}-i \exp [i(k r-\lambda \pi / 2)]
$$

as well as the fact that

$$
\begin{aligned}
\sum_{m} \mathcal{Y}_{m}^{*}\left(\varphi_{r}\right) \mathcal{Y}_{m}\left(\varphi_{k}\right) & = \begin{cases}\frac{1}{2 \pi} & , \quad \ell=0 \\
\frac{1}{2 \pi}\left(e^{-i \ell \varphi_{r}} e^{i \ell \varphi_{k}}+e^{i \ell \varphi_{r}} e^{-i \ell \varphi_{k}}\right) & , \quad \ell>0\end{cases} \\
& =\frac{\epsilon_{\ell}}{2 \pi} \cos \left[\ell\left(\varphi_{k}-\varphi_{r}\right)\right],
\end{aligned}
$$

we can write the asymptotic behaviour of the scattering wave function as

$$
\psi_{\vec{k}}(\vec{r}) \underset{r \rightarrow \infty}{\longrightarrow} \frac{1}{2 \pi}\left[e^{i \vec{k} \vec{r}}+\mathcal{F}(E, \varphi) \frac{e^{i k r}}{\sqrt{r}}\right],
$$

where $\varphi=\varphi_{k}-\varphi_{r}$ is the scattering angle and the total scattering amplitude has the following partial-wave expansion

$$
\mathcal{F}(E, \varphi)=\sum_{\ell=0}^{\infty} \epsilon_{\ell} f_{\ell}(E) \cos (\ell \varphi)
$$

The cross section for a two-dimensional scattering has the units of length. The number of particles scattered into the angle spanned by the arc $r d \varphi$, is the product of the flux in that radial direction and the length of the arc. The corresponding cross section $d \sigma$ is defined as such a length that after its multiplication by the total incoming flux, it gives the same number of particles, i.e.

$$
\left|\vec{j}^{(\text {in })}\right| d \sigma=j_{r}^{\text {(out) }}(\varphi) r d \varphi .
$$

Using standard definition for the particle flux $\vec{j}=\hbar /(2 i \mu)\left(\psi^{*} \vec{\nabla} \psi-\psi \vec{\nabla} \psi^{*}\right)$ with the operator $\vec{\nabla}$ given by Eq. (89), it is not difficult to find that the incoming [corresponding to the first term of the wave function (114)] and outgoing (obtained from the second term of the same wave function) fluxes are

$$
\vec{j}^{(\text {in })}=\frac{\hbar \vec{k}}{(2 \pi)^{2} \mu}, \quad j_{r}^{\text {(out) }}(\varphi)=\frac{\hbar k|\mathcal{F}(E, \varphi)|^{2}}{(2 \pi)^{2} \mu r}
$$

and thus the differential cross section is

$$
\frac{d \sigma}{d \varphi}=|\mathcal{F}(E, \varphi)|^{2} .
$$


Using the integral

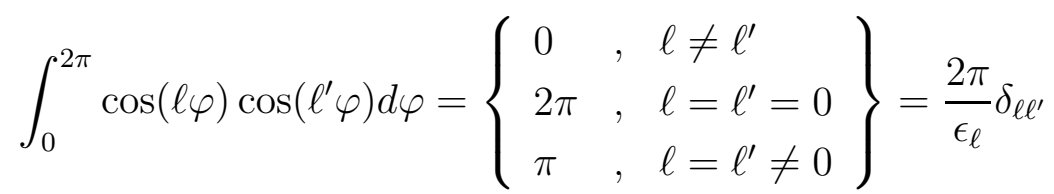

the total cross section can be written as follows

$$
\begin{aligned}
\sigma & =\int_{0}^{2 \pi}|\mathcal{F}(E, \varphi)|^{2} d \varphi=\sum_{\ell} \sigma_{\ell}, \\
\sigma_{\ell} & =2 \pi \epsilon_{\ell}\left|f_{\ell}(E)\right|^{2}=\frac{\epsilon_{\ell}}{k}\left|s_{\ell}(E)-1\right|^{2},
\end{aligned}
$$

where $\sigma_{\ell}$ is the partial-wave cross section.

\section{B Expansion coefficients for the holomorphic parts of the Riccati-Bessel and Riccati-Neumann functions}

The Riccati-Bessel and Riccati-Neumann functions $j_{\lambda}(k r)$ and $y_{\lambda}(k r)$ can be written in the following factorized form

$$
\begin{aligned}
j_{\lambda}(k r) & =k^{\lambda+1} \tilde{j}_{\lambda}(E, r), \\
y_{\lambda}(k r) & =k^{-\lambda} \tilde{y}_{\lambda}(E, r)+k^{\lambda+1} h(k) \tilde{j}_{\lambda}(E, r),
\end{aligned}
$$

where the "tilded" functions are holomorphic with respect to the energy variable $E$. This means that we can expand them in the Taylor series,

$$
\begin{aligned}
& \tilde{j}_{\lambda}(E, r)=\sum_{n=0}^{\infty} s_{n}^{(\lambda)}\left(E_{0}, r\right)\left(E-E_{0}\right)^{n}, \\
& \tilde{y}_{\lambda}(E, r)=\sum_{n=0}^{\infty} c_{n}^{(\lambda)}\left(E_{0}, r\right)\left(E-E_{0}\right)^{n},
\end{aligned}
$$

near an arbitrary point $E_{0}$. The expansion coefficients,

$$
\begin{aligned}
& s_{n}^{(\lambda)}\left(E_{0}, r\right)=\left.\frac{1}{n !} \frac{\partial^{n}}{\partial E^{n}} \tilde{j}_{\lambda}(E, r)\right|_{E=E_{0}}, \\
& c_{n}^{(\lambda)}\left(E_{0}, r\right)=\left.\frac{1}{n !} \frac{\partial^{n}}{\partial E^{n}} \tilde{y}_{\lambda}(E, r)\right|_{E=E_{0}},
\end{aligned}
$$


are expressed via the corresponding derivatives. In order to find them, we notice that

$$
E=\frac{\hbar^{2} k^{2}}{2 \mu} \Longrightarrow \frac{\partial}{\partial E}=\frac{\mu}{\hbar^{2} k} \frac{\partial}{\partial k}
$$

and also make use of the relations (which follow from Eq.(9.1.30) of the handbook by M. Abramowitz and I. A. Stegun)

$$
\begin{aligned}
\frac{d}{d z}\left[\frac{\mathcal{J}_{\lambda}(z)}{z^{\lambda+1}}\right] & =-\frac{\mathcal{J}_{\lambda+1}(z)}{z^{\lambda+1}}, \\
\frac{d}{d z}\left[z^{\lambda} \mathcal{J}_{\lambda}(z)\right] & =z^{\lambda} \mathcal{J}_{\lambda-1}(z),
\end{aligned}
$$

where $\mathcal{J}_{\lambda}(z)$ stands for either $j_{\lambda}(z)$ or $y_{\lambda}(z)$. Therefore

$$
\begin{aligned}
\frac{\partial}{\partial E} \tilde{j}_{\lambda}(E, r) & =\frac{\mu}{\hbar^{2} k} \cdot \frac{\partial}{\partial k}\left[\frac{j_{\lambda}(k r)}{k^{\lambda+1}}\right]=\frac{\mu r^{\lambda+2}}{\hbar^{2} k} \cdot \frac{\partial}{\partial(k r)}\left[\frac{j_{\lambda}(k r)}{(k r)^{\lambda+1}}\right]= \\
& =-\frac{\mu r^{\lambda+2}}{\hbar^{2} k} \frac{j_{\lambda+1}(k r)}{(k r)^{\lambda+1}}=-\frac{\mu r}{\hbar^{2}} \tilde{j}_{\lambda+1}(E, r)
\end{aligned}
$$

and thus

$$
\begin{gathered}
\frac{\partial^{n}}{\partial E^{n}} \tilde{j}_{\lambda}(E, r)=\left(-\frac{\mu r}{\hbar^{2}}\right)^{n} \tilde{j}_{\lambda+n}(E, r), \\
s_{n}^{(\lambda)}\left(E_{0}, r\right)=\frac{1}{n !}\left(-\frac{\mu r}{\hbar^{2}}\right)^{n}\left[\frac{j_{\lambda+n}(k r)}{k^{\lambda+n+1}}\right]_{E=E_{0}}=\frac{1}{n !}\left(-\frac{\mu r}{\hbar^{2}}\right)^{n} \sqrt{\frac{\pi r}{2}}\left[\frac{J_{\ell+n}(k r)}{k^{\ell+n}}\right]_{E=E_{0}} .
\end{gathered}
$$

As it should be (since $\tilde{j}_{\lambda}(E, r)$ is single-valued), the expansion coefficients $s_{n}^{(\lambda)}\left(E_{0}, r\right)$ do not depend on the choice of the sign of the momentum $k_{0}= \pm \sqrt{2 \mu E_{0} / \hbar^{2}}$. Indeed,

$$
\frac{j_{\lambda+n}(k r)}{k^{\lambda+n+1}}=\sqrt{\frac{\pi k r}{2}} \frac{J_{\lambda+n+1 / 2}(k r)}{k^{\lambda+n+1}}=\sqrt{\frac{\pi r}{2}} \frac{J_{\lambda+n+1 / 2}(k r)}{k^{\lambda+n+1 / 2}},
$$

and since (see Eq.(9.1.35) of M. Abramowitz et al.)

$$
J_{\nu}\left(z e^{i \pi}\right)=\left(e^{i \pi}\right)^{\nu} J_{\nu}(z)
$$

the numerator and denominator in Eq. (128) acquire the same phase factor when $k$ changes its sign. 
Finding the derivative $\partial_{E}^{n} \tilde{y}_{\lambda}(E, r)$ is a little bit more complicated. The first derivative can be written as

$$
\frac{\partial}{\partial E} \tilde{y}_{\lambda}(E, r)=\frac{\mu r^{1-\lambda}}{\hbar^{2} k}\left\{\frac{\partial}{\partial(k r)}\left[(k r)^{\lambda} y_{\lambda}(k r)\right]-\frac{\partial}{\partial(k r)}\left[h(k)(k r)^{\lambda} j_{\lambda}(k r)\right]\right\} .
$$

Using Eq. (125) and explicit form of the function $h(k)$ given by Eq. (31), we obtain

$$
\begin{aligned}
\frac{\partial}{\partial E} \tilde{y}_{\lambda}(E, r) & =\frac{\mu r^{1-\lambda}}{\hbar^{2} k}\left[(k r)^{\lambda} y_{\lambda-1}(k r)-h(k)(k r)^{\lambda} j_{\lambda-1}(k r)-(k r)^{\lambda} j_{\lambda}(k r) \frac{2}{\pi k r}\right] \\
& =\frac{\mu r}{\hbar^{2}}\left[k^{\lambda-1} y_{\lambda-1}(k r)-k^{\lambda-1} h(k) j_{\lambda-1}(k r)-\frac{2}{\pi r} k^{\lambda-2} j_{\lambda}(k r)\right] \\
& =\frac{\mu r}{\hbar^{2}} \tilde{y}_{\lambda-1}(E, r)-\frac{2 \mu}{\pi \hbar^{2}} k^{\lambda-2} j_{\lambda}(k r) \\
& =\frac{\mu r}{\hbar^{2}} \tilde{y}_{\lambda-1}(E, r)-\frac{2 \mu}{\pi \hbar^{2}} f_{\lambda 1}(k r),
\end{aligned}
$$

where we introduced an auxiliary function

$$
f_{\lambda n}(k, r)=k^{\lambda-2 n} j_{\lambda}(k r)=k^{\ell-2 n} \sqrt{\frac{\pi r}{2}} J_{\ell}(k r)
$$

whose derivatives can be found using the following recurrence relation

$$
\begin{aligned}
\frac{\partial}{\partial E}\left[k^{\lambda-2 n} j_{\lambda}(k r)\right] & =\frac{\mu}{\hbar^{2} k} r^{2 n-\lambda+1} \frac{\partial}{\partial(k r)}\left[\frac{1}{(k r)^{2 n}}(k r)^{\lambda} j_{\lambda}(k r)\right] \\
& =\frac{\mu}{\hbar^{2} k} r^{2 n-\lambda+1}\left[-\frac{2 n}{(k r)^{2 n+1}}(k r)^{\lambda} j_{\lambda}(k r)+\frac{1}{(k r)^{2 n}}(k r)^{\lambda} j_{\lambda-1}(k r)\right] \\
& =-\frac{2 n \mu}{\hbar^{2}} k^{\lambda-2(n+1)} j_{\lambda}(k r)+\frac{\mu r}{\hbar^{2}} k^{\lambda-1-2 n} j_{\lambda-1}(k r),
\end{aligned}
$$

i.e.

$$
\frac{\partial}{\partial E} f_{\lambda n}=-\frac{2 n \mu}{\hbar^{2}} f_{\lambda, n+1}+\frac{\mu r}{\hbar^{2}} f_{\lambda-1, n}
$$

Repeatedly using the relations (130) and (132), we can calculate any number of the derivatives $\partial_{E}^{n} \tilde{y}_{\lambda}(E, r)$ needed for finding the expansion coefficients (122). 


\section{References}

[1] A. K. Aringazin, Yu. Dahnovsky, V. D. Krevchik, M. B. Semenov, A. A. Ovchinnikov, K. Yamamoto, "Two-dimensional tunnel correlations with dissipation", Phys.Rev., B $68,155426(2003)$

[2] S. A. Rakityansky, "Unified treatment of bound, scattering, and resonant states in one-dimensional semiconductor nanostructures", Phys. Rev. B 68, 195320 (2003)

[3] S. A. Rakityansky, " Modified transfer matrix for nanostructures with arbitrary potential profile", Phys. Rev. B 70, 205323 (2004)

[4] D. Bollé, F. Gesztesy, "Low-energy parametrization of scattering observables in $n$ dimensional quantum systems", Phys. Rev. Lett., 52, 1469 (1984)

[5] D. Bollé, F. Gesztesy, "Scattering observables in arbitrary dimension $n \geqslant 2$ ", Phys. Rev., A30, 1279 (1984)

[6] B. J. Verhaar, J. P. H. W. van den Eijnde, M. A. J. Voermans, M. M. J. Schaffrath, "Scattering length and effective range in two dimensions; application to adsorbed hydrogen atoms", J. Phys. A: Math. Gen., 17, 595 (1984)

[7] B. J. Verhaar, L. P. H. De Goey, E. J. D. Vredenbregt, J. P. H. W. van den Eijnde, "Scattering length and effective range for scattering in a plane and in higher dimensions", Phys. Lett., A110, 371 (1985)

[8] B. J. Verhaar, L. P. H. De Goey, E. J. D. Vredenbregt, J. P. H. W. van den Eijnde, "Scattering length and effective range for scattering in a plane and in higher dimensions", Phys. Rev., A32, 1424 (1985)

[9] W. G. Gibson, " Two-dimensional scattering: low-energy behaviour of the Jost function and Levinson's theorem", Phys. Lett., A117, 107 (1986)

[10] M. Klawunn, A. Pikovski, L. Santos, " Two-dimensional scattering and bound states of polar molecules in bilayers", Phys.Rev., A 82, 044701 (2010)

[11] H. A. Bethe, "Theory of the Effective Range in Nuclear Scattering", Phys. Rev., 76, 38-50 (1949)

[12] K. Helfrich and H.-W. Hammer, "Resonant three-body physics in two spatial dimensions", arXiv: 1101.1891v1[cond-mat.quant-gas](2011) 
[13] S. A. Rakityansky, N. Elander, "Generalized effective-range expansion", J. Phys. A: Math. Theor. 42, 225302 (2009)

[14] S. A. Rakityansky, N. Elander, "Multi-channel analog of the effective-range expansion", J. Phys. A: Math. Theor. 44, 115303 (2011)

[15] L. Brand, "Differential and Difference Equations", (Wiley, New York), (1966)

[16] Mathews J and Walker L R 1964 " Mathematical Methods of Physics" (New York: Benjamin)

[17] Abramowitz M and Stegun A (ed), "Handbook of Mathematical Functions", Washington, DC: National Bureau of Standards (1964)

[18] Rakityansky S A, Sofianos S A and Amos K 1996 A method of calculating the Jost function for analytic potentials Nuovo Cimento B 11136378

[19] Sofianos S A and Rakityansky S A 1997 Exact method for locating potential resonances and Regge trajectories J. Phys. A: Math. Gen. 303725

[20] Sofianos S A, Rakityansky S A and Vermaak G P 1997 Sub-threshold resonances in few-neutron systems J.Phys. G: Nucl. Part. Phys. 23161929

[21] Rakityansky S A and Sofianos S A 1998 Jost function for coupled partial waves J. Phys. A: Math. Gen. 31514975

[22] Sofianos S A, Rakityansky S A and Massen S E 1999 Jost function for singular potentials Phys. Rev. A 6033743

[23] Rakityansky S A and Sofianos S A 1999 Jost function for coupled channels Few-Body Syst. Suppl. 10936

[24] Massen S E, Sofianos S A, Rakityansky S A and Oryu S 1999 Resonances and off-shell characteristics of effective interactions Nucl. Phys. A 654597611

[25] Rakityansky S A, Sofianos S A and Elander N 2007 Pade approximation of the $S$-matrix as a way of locating quantum resonances and bound states J. Phys. A: Math. Theor. 401485769

[26] H. Poincaré, "Sur les groupes des équations linéaires", Acta Mathematica, 4, 201-311 (1884).

See also: Lefschetz S 1957 Differential Equations: Geometric Theory (New York: Interscience) 
[27] Motovilov A K 1993 Theor. Math. Phys. 95692

[28] A. J. Markvoort, P. A. Hilbers, R. Pino, " Laterally coupled jellium-like two-dimensional quantum dots", J. Phys.: Condens. Matter, 15, 6977, (2003)

[29] S. De Filippo, M. Salerno, "Spectral properties of a model potential for quantum dots with smooth boundaries", Phys. Rev. B, 62, 4230, (2000)

[30] J. Adamowski, M. Sobkowicz, B. Szafran, S. Bednarek, "Electron pair in a Gaussian confining potential', Phys. Rev. B, 62, 4234, (2000)

[31] O. Ciftja, "An experimentally justified confining potential for electrons in twodimensional semiconductor quantum dots", Journal of Computer-Aided Material Design, 14, 37, (2007)

[32] M. Cheney, " Two-dimensional scattering: The number of bound states from scattering data", J. Math. Phys., 25(5), pp. 1449-1455 (1984)

[33] D. Bollé, F. Gesztesy, C. Danneels, S. F. J. Wilk, " Threshold Behavior and Levinson's Theorem for Two-Dimensional Scattering Systems: A Surprise", Phys. Rev. Lett. 56, pp. 900-903 (1986)

[34] I. R. Lapidus, "Quantum-mechanical scattering in two dimensions", Am.J.Phys., 50, $45(1982)$

[35] S. K. Adhikari, "Quantum scattering in two dimensions", Am.J.Phys., 54, 362 (1986)

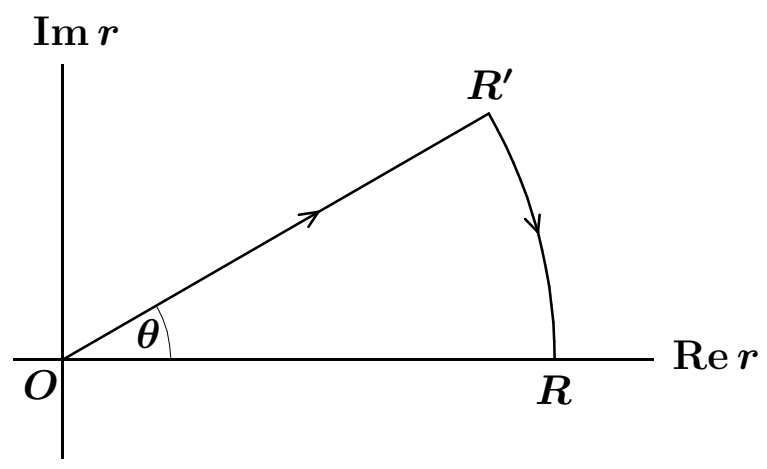

Figure 1: Deformed contour for integrating differential equations (14|15) and (22||23). 


\begin{tabular}{|r|c|}
\hline \multicolumn{1}{|c|}{$E_{r}$} & $\Gamma$ \\
\hline-32.4850428093 & 0 \\
\hline-16.2643650096 & 0 \\
\hline-6.2711504590 & 0 \\
\hline 0.5036180960 & $2 \times 10^{-15}$ \\
\hline 4.9422440057 & 0.0000588188 \\
\hline 7.1050168573 & 0.5710776714 \\
\hline 7.9987409699 & 3.6684977768 \\
\hline 8.5025637363 & 7.7605743107 \\
\hline 8.5937554145 & 12.3052581635 \\
\hline 8.3193121385 & 17.0922638769 \\
\hline 7.6952969586 & 21.9663916836 \\
\hline 6.7436612278 & 26.9367555304 \\
\hline 5.5244040747 & 31.8688591621 \\
\hline 4.0100103640 & 36.8118853195 \\
\hline 2.2603614329 & 41.6490284540 \\
\hline
\end{tabular}

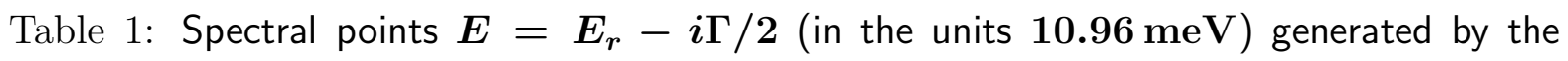
potential (84). Their distribution on the complex energy surface is shown in Fig. 3]. 


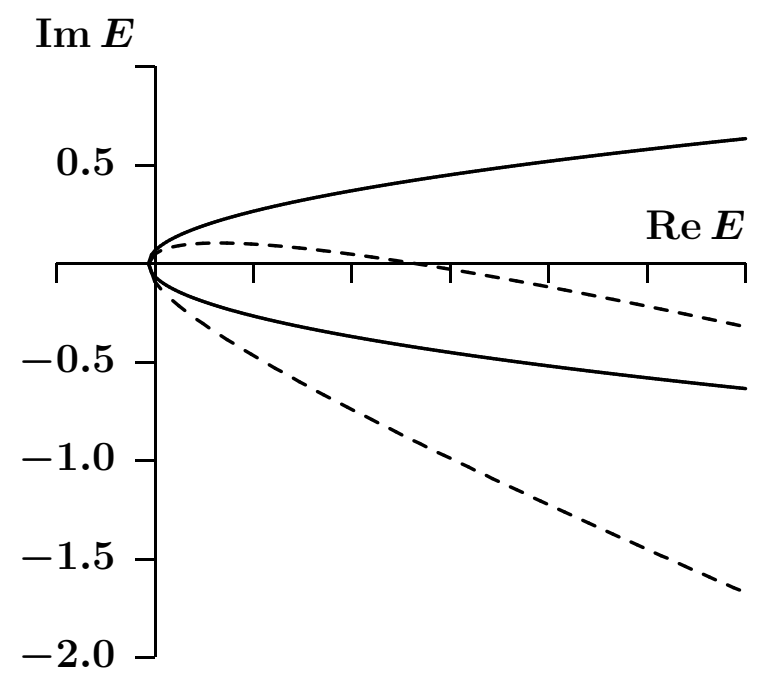

Figure 2: Domains $\mathcal{D}$ for the potential (84), defined by Eq. (51) (within the solid curve) and by Eq. (53) for the rotation angle $\theta=0.05 \pi$ (within the dashed curve). The energy is given in the donor Hartree units (see Sec. 9).

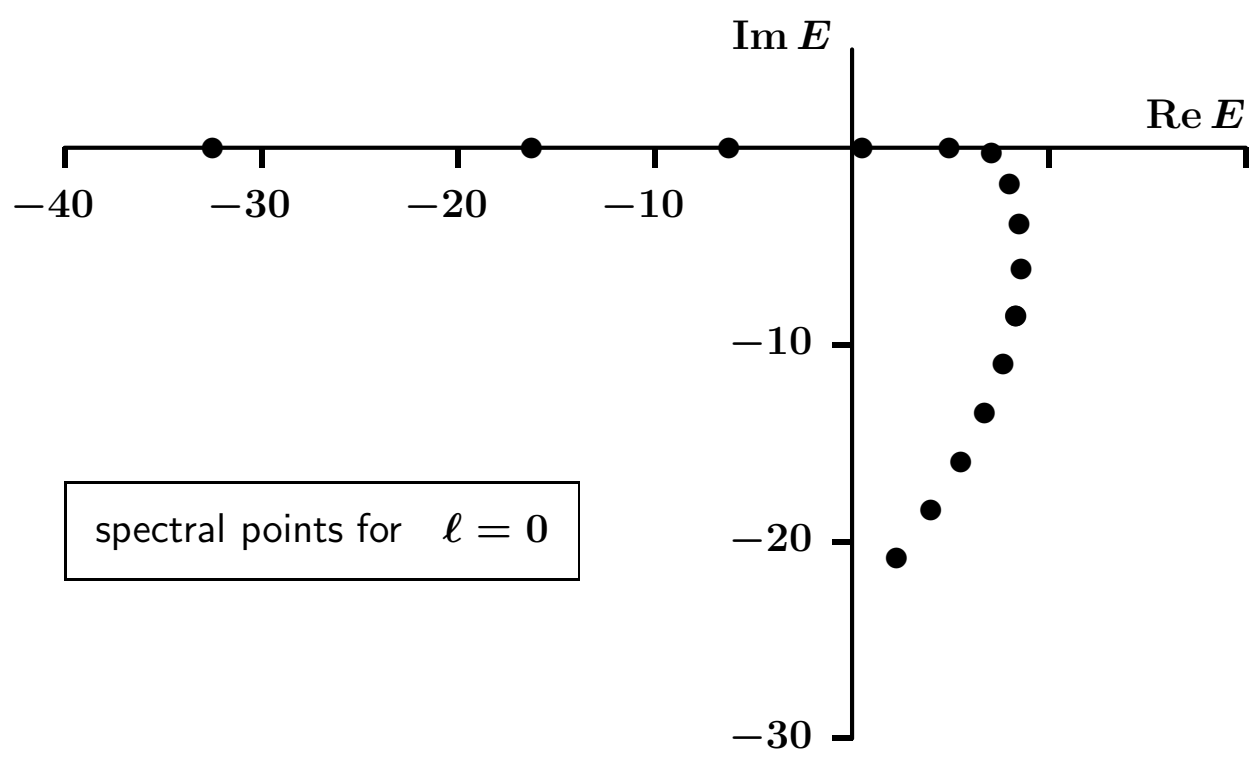

Figure 3: Spectral points generated by the potential (84). Their numerical values are given in Table 1 . 


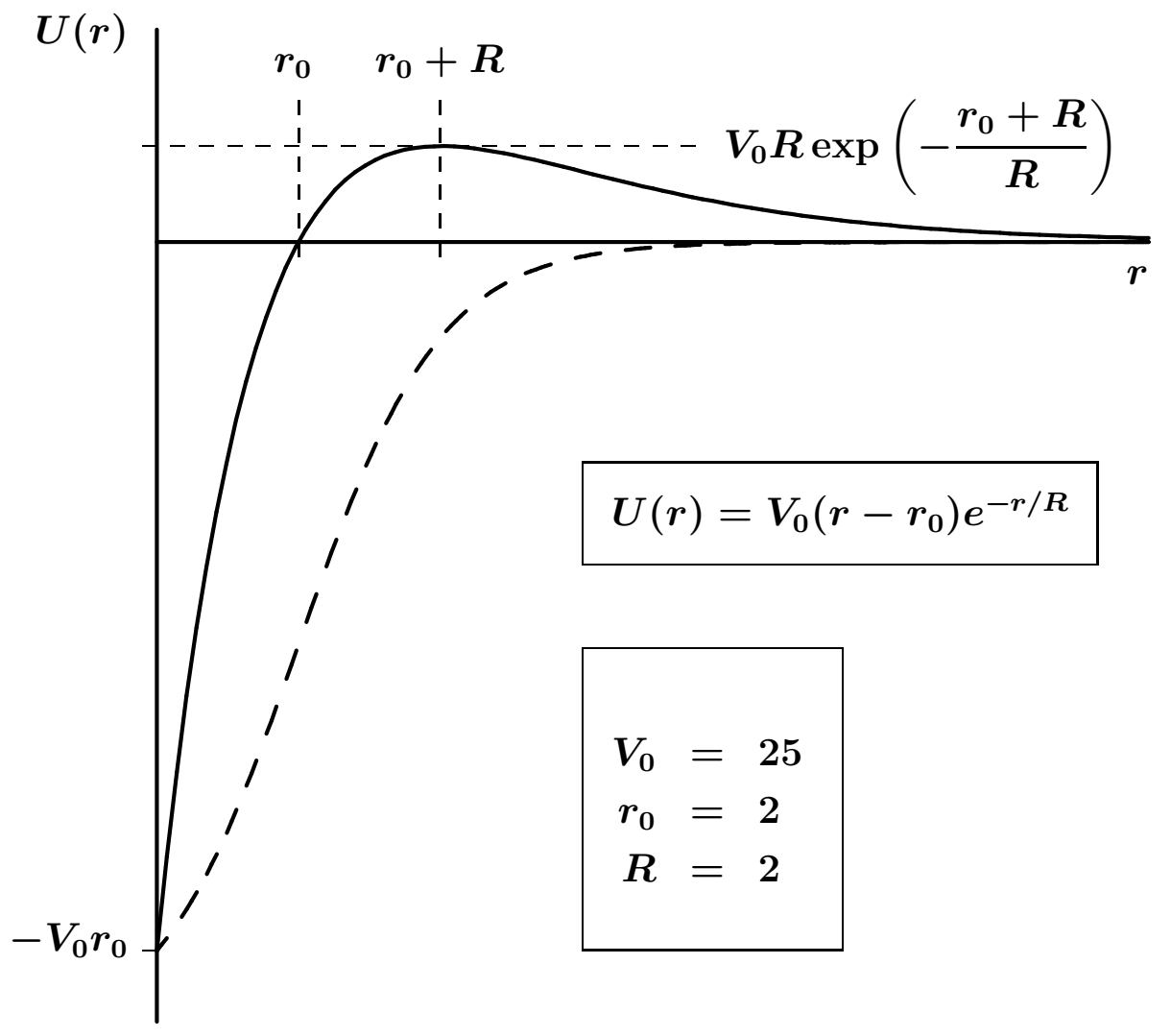

Figure 4: Model potential (84) measured in the donor Hartree units $10.96 \mathrm{meV}$ as a function of the distance measured in the units of donor Bohr radius $101.89 \AA$. The dashed curve is a typical potential for an empty two-dimensional quantum dot. 


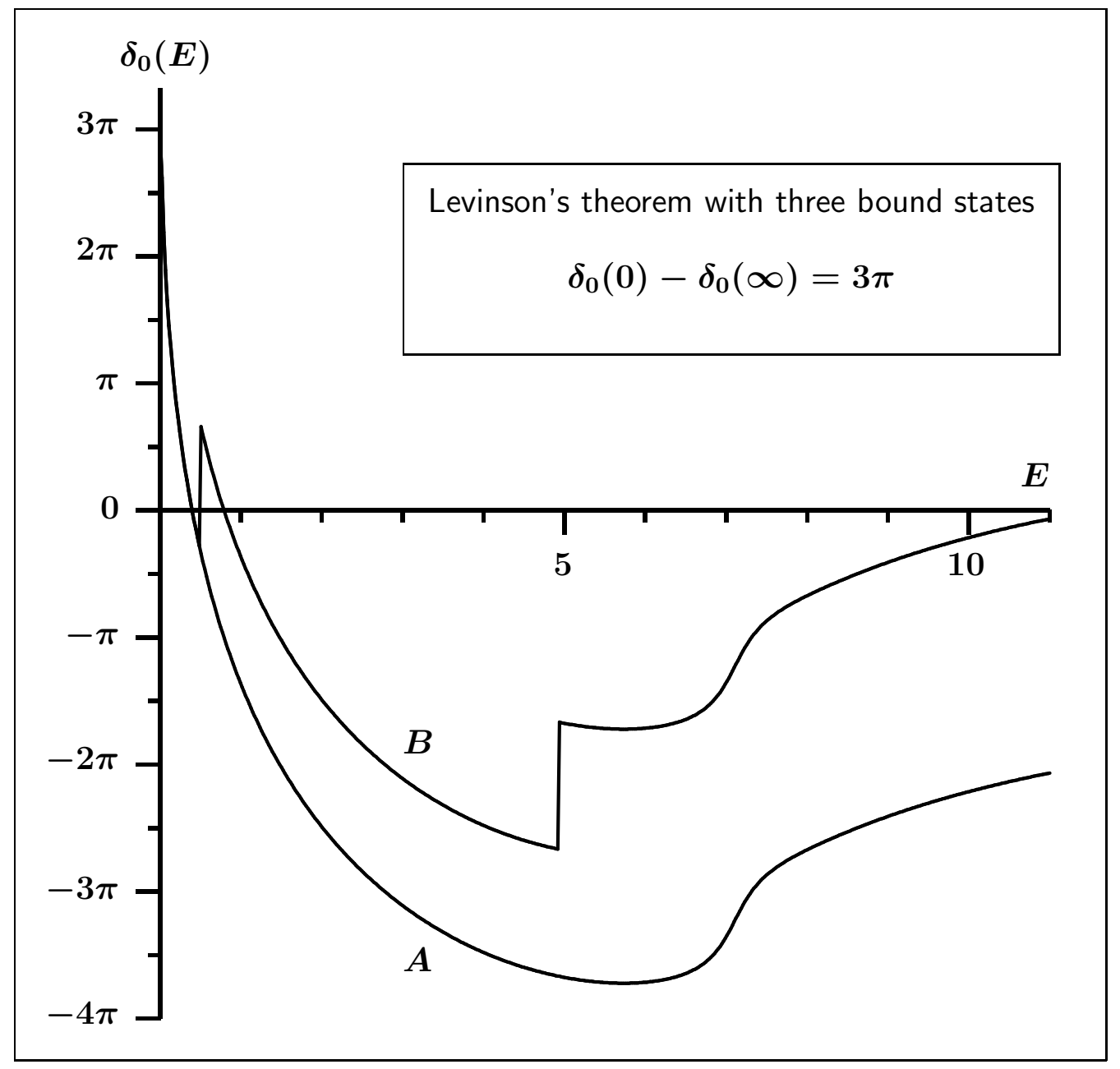

Figure 5: $S$-wave scattering phase-shift for the potential (84). In the curve "A" the sharp jumps in $\pi$ (corresponding to the first two extremely narrow resonances) are missing and as a result it does not obey the Levinson's theorem. 


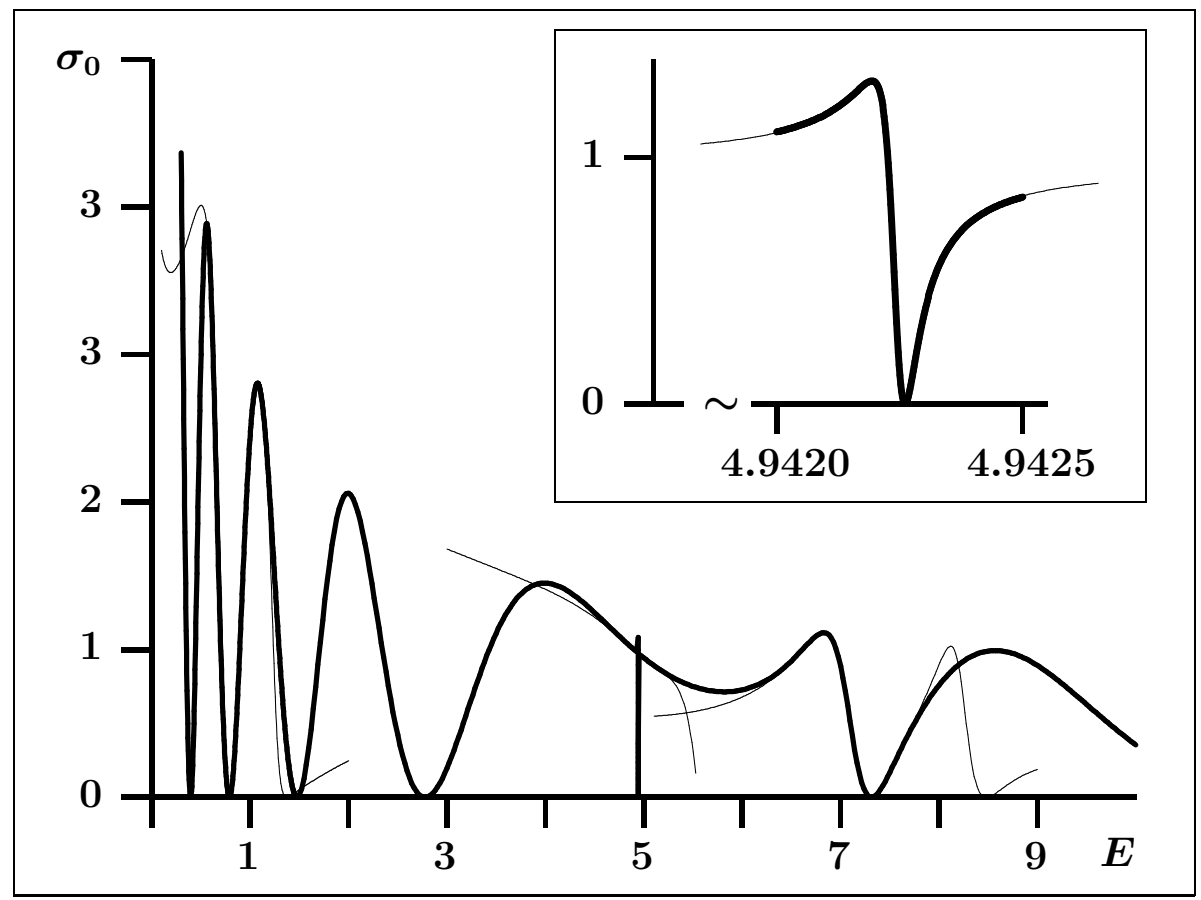

Figure 6: Comparison of the exact (thick curve) and approximate (thin curve) $S$-wave cross section for the potential (84). The approximate curves are obtained using the expansion (69) with $N=4$ (five terms) near the points $E_{0}=1 \times 10.96 \mathrm{meV}, E_{0}=5 \times 10.96 \mathrm{meV}$, and $E_{0}=7 \times 10.96 \mathrm{meV}$. The insert shows a magnified fragment of the curves near the second resonance. 


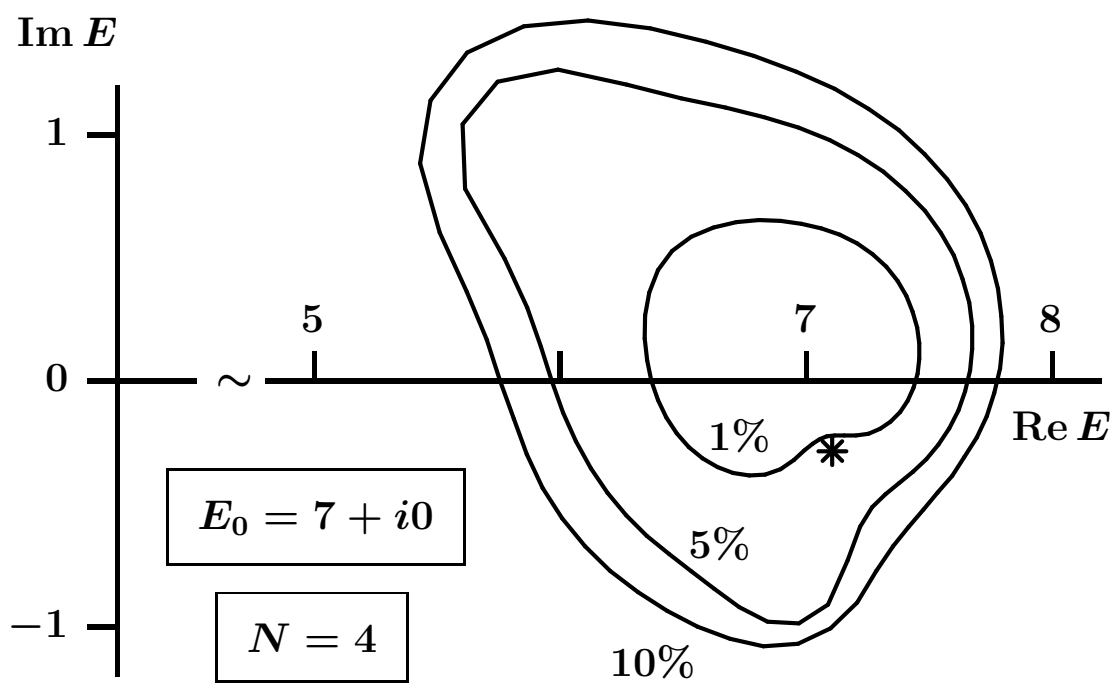

Figure 7: The domains within which the Jost function for the potential (84) is reproduced, using the first five terms $(N=4)$ of the expansion (69), with the accuracy better than $1 \%$, $5 \%$ and $10 \%$. The expansion is done around the point $E_{0}=7 \times[10.96 \mathrm{meV}]$ on the real axis. The star shows the third resonance given in Table 1 .

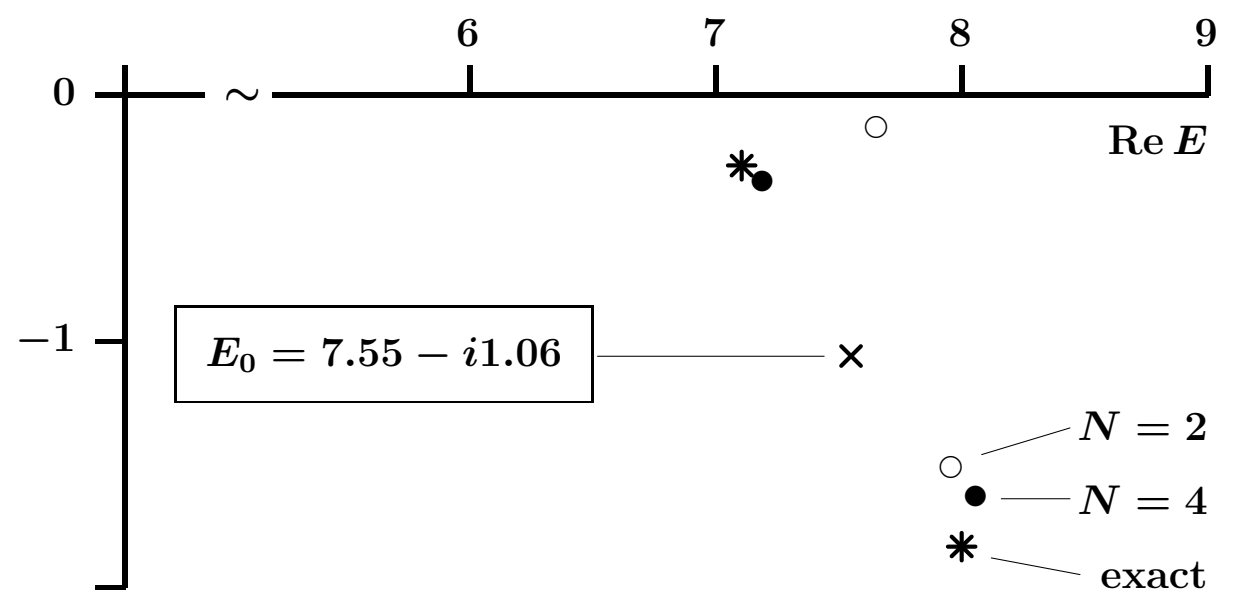

$\operatorname{Im} E$

Figure 8: Spectral points corresponding to the third and fourth resonances of the potential (84). Stars show their exact locations. Open and filled circles are obtained using the expansion (69) with $N=2$ (three terms) and $N=4$ (five terms), respectively. The expansion is done around the point $E_{0}=(7.55-i 1.06) \times 10.96 \mathrm{meV}$, which is in the middle between these two resonances. 


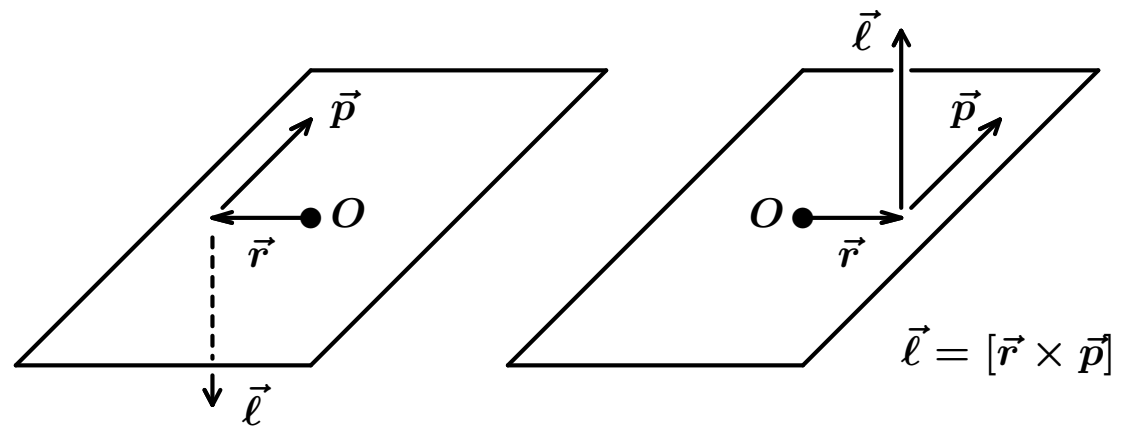

Figure 9: Two possible directions of the angular momentum for a particle moving on a plane. 